AEI-2010-120

August 2010

\title{
$(0,2)$ Elephants
}

\author{
Paul S. Aspinwall ${ }^{1}$, Ilarion V. Melnikov ${ }^{2}$ and M. Ronen Plesser ${ }^{1}$ \\ ${ }^{1}$ Center for Geometry and Theoretical Physics, Box 90318 \\ Duke University, Durham, NC 27708-0318 \\ ${ }^{2}$ Max-Planck-Institut für Gravitationsphysik (Albert-Einstein-Institut), \\ Am Mühlenberg 1, D-14476 Golm, Germany
}

\begin{abstract}
We enumerate massless $\mathrm{E}_{6}$ singlets for $(0,2)$-compactifications of the heterotic string on a Calabi-Yau threefold with the "standard embedding" in three distinct ways. In the large radius limit of the threefold, these singlets count deformations of the Calabi-Yau together with its tangent bundle. In the "small-radius" limit we apply Landau-Ginzburg methods. In the orbifold limit we use a combination of geometry and free field methods. In general these counts differ. We show how to identify states between these phases and how certain states vanish from the massless spectrum as one deforms the complex structure or Kähler form away from the Gepner point. The appearance of extra singlets for particular values of complex structure is explored in all three pictures, and our results suggest that this does not depend on the Kähler moduli.
\end{abstract}




\section{Introduction}

The earliest form of model building in string theory consisted of "embedding the spin connection in the gauge group" for a Calabi-Yau compactification of the $\mathrm{E}_{8} \times \mathrm{E}_{8}$ heterotic string [1]. These correspond to (0,2)-compactifications that happen to have $N=(2,2)$ worldsheet supersymmetry. A natural question to ask concerns the counting of massless states in uncompactified spacetime which are singlets under the unbroken $\mathrm{E}_{8} \times \mathrm{E}_{6}$ gauge symmetry. This turns out to be a fascinating question that has received rather sporadic attention in the past 25 years.

These massless states correspond to first order deformations of the theory. Marginal deformations must preserve the $(0,2)$ superconformal symmetry [2]. Deformations that preserve the full $(2,2)$ invariance constitute the familiar $(2,2)$ moduli space. Its dimension is constant [3], and in a geometric phase it corresponds to the unobstructed [4] deformations of complex structures and changes in the complexified Kähler form. The remaining moduli that only preserve $(0,2)$ invariance are harder to describe. As a first step, we may count the massless gauge singlets in the four-dimensional effective theory. Each of these is a first order deformation that may be obstructed at higher order.

Unfortunately, the identification of all massless singlets at a generic point in the $(2,2)$ moduli space is well beyond our current abilities. To make progress, we must work at certain limiting points where the spectrum is accessible to available techniques. These include large radius points, Landau-Ginzburg loci and the Gepner points they contain, and orbifolds. In each of these points the techniques used to identify the singlets are rather different, and the resulting description of the space of first order deformations might appear as mysterious as an elephant to the group of proverbial blind men from Indostan. Can these different descriptions be reconciled?

In the large radius limit of a Calabi-Yau phase, the counting of the singlets is quite easy to visualize. The $(2,2)$ singlets manifest themselves as infinitesimal deformations of the complex structure or complexified Kähler form of the Calabi-Yau, while the less familiar $(0,2)$ singlets correspond to first order deformations of the tangent bundle, counted by $H^{1}($ End $T)$. This group can jump with complex structure [5]; moreover a "generic" first order $(0,2)$ deformation is expected to be lifted by world-sheet instantons [6]. One might expect, therefore, that the Gepner models corresponding to certain Calabi-Yau threefolds might count the number of (0,2)-deformations differently. After all, the Gepner model describes physics at some "minimal radius" for the Calabi-Yau threefold, well away from the large radius limit, for a special choice of complex structure. Singlet counts for Gepner models were comprehensively listed in [7].

So perhaps the different aspects of the elephant cannot be reconciled. It may be that the number of singlets varies wildly across the moduli space. We will argue here that is not the case. The behaviour of the singlets is quite orderly, with relatively modest jumping in the singlet count. As is well studied, the quintic threefold provides a remarkably boring case study, where the number of singlets is fixed except for a handful of singlets associated with extra $\mathrm{U}(1)$ gauge symmetries at the Gepner point.

In the case of the quintic, the gauged linear sigma model offers a beautiful explanation of 
this behaviour. As already noted in [8], the $(2,2)$ GLSM describing a Calabi-Yau complete intersection in a toric variety has natural $(0,2)$-preserving deformations encoded in a $(0,2)$ superpotential for the gauge theory. The holomorphic parameters of this superpotential encode the "toric" Kähler moduli, the "polynomial" complex structure moduli, as well as a subset of classically unobstructed bundle moduli. This GLSM parameter space was recently studied in some detail in the case of Calabi-Yau hypersurfaces [9]. Remarkably, these GLSM deformations have been argued to correspond to exactly marginal deformations of the $(0,2)$ theory $10-12]$.

Getting back to the quintic, it is not hard to see that all elements of $H^{1}(\operatorname{End} T)$ can be represented as deformations of the $(0,2)$ GLSM superpotential. Hence, it is not too surprising that the singlet spectrum at the Gepner point simply differs by a few states associated to the un-Higgsing of additional gauge symmetries.

More generally, there are certainly models where $H^{1}(\operatorname{End} T)$ is not fully described by the $(0,2)$ GLSM, and the additional singlets, unprotected by GLSM arguments, should suffer the fate of the "generic" large radius singlet and become massive away from the large radius limit.

In this paper we will study various cases which have a little more structure than the quintic. We will show how the bulk of the spectrum stays fixed and can be tracked nicely between the Calabi-Yau and Landau-Ginzburg pictures. We will also see how various massless states can appear in some subspaces of the moduli space. In some cases these extra states can be tracked all the way from the Gepner model to the large radius limit.

The orbifold is an important intermediate step on a path from the Gepner model to the large radius limit. Comparing the orbifold to the large radius limit is extremely well studied in the context of $(2,2)$ theories. Here the relationship between the orbifold and its resolution is now generally known as the McKay correspondence. A McKay correspondence for the $(0,2)$-case has been quite neglected, despite its origin in string theory being as old [13]. We make some first efforts in this direction here. In particular, at the orbifold limit the massless states can be characterised as "untwisted" or "twisted," and we are able to compute the spectrum of states of both types. In the case of a Calabi-Yau space with a curve of quotient singularities this involves understanding how the six-dimensional theory determined by the quotient is compactified on the singular curve and leads to a twisted compactification familiar from the study of wrapped D-branes. This is a first step toward a McKay correspondence, but there are some subtleties we do not resolve here.

We will focus on 4 examples of Calabi-Yau threefolds in a (weighted) projective space, each of which has its own merit:

- a quintic in $\mathbb{P}^{4}$ is the simplest and most studied case;

- a sextic in $\mathbb{P}_{21111}^{4}$ has extra singlets states at small radius;

- a septic in $\mathbb{P}_{31111}^{4}$ is a blown-up orbifold and demonstrates the $(0,2)$ McKay correspondence;

- an octic in $\mathbb{P}_{22211}^{4}$ exhibits many complications, including extra singlets appearing both at special radii and at special complex structure. 
The Landau-Ginzburg locus for the sextic and octic theories has additional singlets in comparison to the large radius computation, which are not associated with an enhanced gauge symmetry. What is the fate of these singlets as we move away from the LandauGinzburg locus by turning on a Kähler deformation? The only reasonable possibility is that they acquire a Kähler-dependent mass term, which is indeed allowed by the quantum symmetry of the Landau-Ginzburg orbifold and consistent with the fact that the number of additional chiral singlets is even.

This would be challenging to verify directly even at the Gepner point, since it would require us to compute correlators of several twisted states. Luckily, we have a tool at our disposal that would be singularly unhelpful to the six blind men: we can take a look at our elephant in the mirror. Using mirror symmetry we are able to show that the extra singlets do indeed acquire a Kähler-dependent mass.

The extra singlets provide examples of states with Kähler dependent masses; however we observe that in all of our examples every large radius singlet, whether it is a $(0,2)$ GLSM deformation or not, remains massless at the Landau-Ginzburg locus. Thus, we have yet to find an example of a "generic" large radius singlet that is lifted by world-sheet instantons. Instantons could well lead to higher order obstructions for these first order $(0,2)$ deformations, but we find it remarkable that an instanton-induced mass term for the non-GLSM singlets, while allowed by symmetries, is not generated. This suggests that there may be a nonrenormalization theorem with a wider applicability than the one currently known for the subspace of GLSM deformations?

The rest of the article is organized as follows: in sections 2 and 3 we review and develop the technology necessary to study heterotic spectra in Landau-Ginzburg and Calabi-Yau phases. Section 4 is devoted to a comparison of the general results, while section 5 contains specific computations in the examples.

\section{Singlet Spectrum at the Landau-Ginzburg Locus}

Describing the massless spectrum of a heterotic vacuum as a function of the moduli is a difficult affair even in string perturbation theory, since it requires a knowledge of the marginal operators in a non-trivial SCFT. At certain points in the moduli space the SCFT may reduce to a solvable theory: for instance, it might be an orbifold of a free theory or a Gepner model. When this holds, the full perturbative string theory is under control: the spectrum is computable, and any scattering amplitude can be reduced to an integral over the moduli space of a punctured Riemann surface. In principle, conformal perturbation theory can then be used to determine these properties in an open neighborhood of the solvable point. Unfortunately, in practice conformal perturbation theory is difficult to carry out in full generality. For instance, even in an orbifold of $T^{6}$ little is known about the dependence of

\footnotetext{
${ }^{1}$ We have a hint of this possibility already from the $(2,2)$ moduli space: a generic Calabi-Yau hypersurface will have non-toric and non-polynomial deformations that are not described by familiar holomorphic couplings in the GLSM Lagrangian; although standard GLSM arguments do not apply to these, each such deformation corresponds to a deformation of the CFT.
} 
spectrum and amplitudes on the twisted sector moduli corresponding to Kähler deformations.

To make progress it turns out to be useful to sacrifice a little of the ambition: if we cannot determine the spectrum as a function of all the moduli, perhaps we can do so on some suitably nice locus in the moduli space. For instance, in the example of a $T^{6}$ orbifold we can look at the dependence of the spectrum on all of the untwisted moduli. This example is perhaps not very exciting, since as far as this dependence is concerned, we are basically dealing with a solvable theory. The Landau-Ginzburg models provide an important class of examples where the massless spectrum can be determined, even though the theory is not a solvable SCFT. In particular, the Landau-Ginzburg description allows us to follow the massless spectrum as parameters in the superpotential are varied.

\section{$2.1(2,2)$ Landau-Ginzburg Generalities}

In this section we review some standard results on (2,2) Landau-Ginzburg models [14, 15] and their uses in heterotic compactification [16]. A $(2,2)$ Landau-Ginzburg theory with a UV R- symmetry is defined by a Lagrangian for $N$ chiral superfields $X^{i}$ with canonical kinetic terms and a quasi-homogeneous superpotential $W(X)$ satisfying

$$
W\left(\lambda^{\alpha_{i}} X^{i}\right)=\lambda W(X) .
$$

The superpotential coupling is a relevant deformation of the free theory, and under suitable conditions the IR fixed point is believed to be a non-trivial compact $(2,2)$ SCFT, with the UV R-symmetry corresponding to the R-symmetry of the IR theory. In such theories the critical point set of $W$, i.e. points where $d W=0$, is the origin in $\mathbb{C}^{n}$, and without loss of generality the $\alpha_{i}$ may be taken to be $0<\alpha_{i} \leq \frac{1}{2}$.

While following the Landau-Ginzburg RG flow is a non-trivial affair, it turns out that a number of properties of the theory, in particular those involving the supersymmetric ground states, are independent of the RG details. Perhaps the most elegant way to encapsulate the accessible IR physics is via a representation of the left-moving $N=2$ super-conformal algebra in the cohomology of the right-moving supercharge $\bar{Q}$ of the UV theory [17]. The propagating fields in a $(2,2)$ chiral multiplet $X^{i}$ and its complex conjugate anti-chiral multiplet $\bar{X}^{i}$ consist of the bosonic $x^{i}$, its complex conjugate $\bar{x}^{i}$, the right-moving fermions $\psi_{+}^{i}, \bar{\psi}_{+}^{i}$, and the left- 


\begin{tabular}{|c|c|c|c|c|}
\hline & $x^{i}$ & $\partial \bar{x}^{i}$ & $\gamma^{i}$ & $\bar{\gamma}^{i}$ \\
\hline$q$ & $\alpha_{i}$ & $-\alpha_{i}$ & $\alpha_{i}-1$ & $1-\alpha_{i}$ \\
\hline $2 h$ & $\alpha_{i}$ & $2-\alpha_{i}$ & $1+\alpha_{i}$ & $1-\alpha_{i}$ \\
\hline $\bar{q}$ & $\alpha_{i}$ & $-\alpha_{i}$ & $\alpha_{i}$ & $-\alpha_{i}$ \\
\hline
\end{tabular}

Table 1: Weights and charges of the fields

moving fermions $\gamma_{-}^{i}, \bar{\gamma}_{-}^{i}$. Define $T, J, G^{ \pm}$by $\left.\right|^{2}$

$$
\begin{aligned}
T^{0} & =\sum_{i}\left\{-\gamma^{i} \partial \bar{\gamma}^{i}-2 \partial \bar{x}^{i} \partial x^{i}\right\}, \\
J & =\sum_{i}\left\{\left(\alpha_{i}-1\right) \gamma^{i} \bar{\gamma}^{i}-2 \alpha_{i} x^{i} \partial \bar{x}^{i}\right\}, \\
T & =T^{0}-\frac{1}{2} \partial J, \\
G^{-} & =2 i \sqrt{2} \sum_{i} \gamma^{i} \partial \bar{x}^{i}, \\
G^{+} & =i \sqrt{2} \sum_{i}\left\{\left(1-\alpha_{i}\right) \bar{\gamma}^{i} \partial x^{i}-\alpha_{i} x^{i} \partial \bar{\gamma}^{i}\right\} .
\end{aligned}
$$

It is easy to show that these operators are $\bar{Q}$-closed up to the equations of motion of the UV theory; moreover, the super-renormalizability of the Lagrangian allows us to evaluate the algebra of these operators via free-field OPEs. The result is that this is a representation of the $N=2$ algebra with central charge $c=3 \sum_{i}\left(1-2 \alpha_{i}\right)$. The conformal weights $h$ and charges $q$ of the fundamental fields are given in table 2.1; the table also lists the charges $\bar{q}$ under the right-moving R-symmetry.

The left-moving $N=2$ algebra in $\bar{Q}$ cohomology is perfectly suited to study supersymmetric ground states of the theory. In string theory, these right-moving Ramond sector states correspond to massless fermions in the space-time theory, and in a space-time supersymmetric compactification knowledge of these states is sufficient to reconstruct the massless spectrum. While in general determining the $\bar{Q}$ cohomology is a challenge, the superrenormalizability of the Landau-Ginzburg theory reduces the computation to two simple steps [16]: the UV theory is truncated to the fields in the $N=2$ algebra of (1), and the $\bar{Q}$ operator acts on the remaining modes via

$$
\bar{Q}=\oint \frac{d z}{2 \pi i} \gamma^{i} \partial_{i} W(x)
$$

\subsection{The Landau-Ginzburg Orbifold}

In order to apply these ideas to string compactifications, they must be generalized to LandauGinzburg orbifolds. This has been carried out in [16] and extended to a wide class of $(0,2)$ heterotic backgrounds in $[18 \sqrt[20]{ }$. In this section we will review the $(2,2)$ heterotic models.

\footnotetext{
${ }^{2}$ We use the notation where \pm denotes R-charge, not world-sheet chirality.
} 
Following Gepner [21], we know that a $c=\bar{c}=9(2,2)$ SCFT with integral $q-\bar{q}$ charges can be used to construct a space-time supersymmetric $\mathrm{E}_{6} \times \mathrm{E}_{8}$ heterotic compactification. In Landau-Ginzburg models the natural way to achieve this is to orbifold by $\exp (2 \pi i J)[22]$. In the Gepner construction, the "internal" theory is tensored with a free theory of 10 left-moving Majorana-Weyl fermions $\lambda^{A}$, as well as a level-one left-moving $\mathrm{E}_{8}$ current algebra. Adding four $(0,1)$ superfields for the four Minkowski directions leads to correct central charges for a critical heterotic string, and modular invariance and space-time supersymmetry are ensured by performing a GSO projection on the world-sheet fermion numbers on the left and the right. The right-moving GSO projection is, as usual, responsible for space-time supersymmetry, while the left-moving projection ensures that the manifest $\mathrm{SO}(10) \times \mathrm{U}(1)$ gauge symmetry is enhanced to $\mathrm{E}_{6} \cdot{ }^{3}$

In the $(2,2)$ Landau-Ginzburg theory, the GSO projections are conveniently combined with the orbifold onto integral $q$ : we simply orbifold by $g=\exp (-i \pi J)$ and project onto suitable fermion numbers, both on the left and right, mod 2. The massless fermions that are $\mathrm{E}_{6} \times \mathrm{E}_{8}$ singlets come from the (NS, R) sectors, i.e. the sectors twisted by $g^{k}$ with $k$ odd. In such a sector the GSO projection amounts to keeping states with integral $q$. Since $\alpha_{i}$ are rational, $g^{2 d}=1$ for some integer $d$, and hence there are $2 d-1$ twisted sectors. Space-time CPT exchanges the $k$-th twisted sector with the $(2 d-k)$-th sector, so we need only consider $k=1,3, \ldots, d$.

Massless fermion states must be in the $\bar{Q}$ cohomology, and level matching implies that the left-moving energy $E$ must vanish. The $\mathrm{E}_{6}$ representation, as well as the type of space-time multiplet (vector or chiral/anti-chiral) is determined by the $q, \bar{q}$ charges. The $\mathrm{E}_{6}$ representation follows from the standard decomposition

$$
\begin{aligned}
& \mathrm{E}_{6} \mapsto \mathrm{SO}(10) \times \mathrm{U}(1) \\
& \mathbf{7 8} \mapsto \mathbf{4 5}_{0}+\mathbf{1 6}_{-3 / 2}+\overline{\mathbf{1 6}}_{3 / 2}+\mathbf{1}_{0} \\
& \mathbf{2 7} \mapsto \mathbf{1 6}_{1 / 2}+\mathbf{1 0}_{-1}+\mathbf{1}_{2} \\
& \overline{\mathbf{2 7}} \mapsto \overline{\mathbf{1 6}}_{-1 / 2}+\mathbf{1 0}_{1}+\mathbf{1}_{-2} .
\end{aligned}
$$

The $\mathrm{E}_{6}$ singlet states must have $q=0$. The type of space-time multiplet can be determined by working out the action of spectral flow on corresponding (NS,NS) operators. The result is that a massless fermion with right-moving charge $\bar{q}= \pm \frac{3}{2}$ is a vector; if $\bar{q}=-\frac{1}{2}$ it belongs to a chiral multiplet, and if $\bar{q}=\frac{1}{2}$, it belongs to an anti-chiral multiplet.

The algorithm for determining the singlet spectrum is therefore quite simple: for each odd sector in the Landau-Ginzburg orbifold, we must identify states in the $\bar{Q}$ cohomology with $E=q=0$ and $\bar{q}= \pm \frac{1}{2}$. Since we restrict to the $\bar{Q}$ cohomology the left-moving quantum numbers can be determined from the left-moving $N=2 \mathrm{UV}$ algebra. It is useful to note that $\bar{Q}$ commutes with the left-moving algebra and has a definite right-moving charge $\bar{q}=1$. Thus, in any sector the zero energy states live in a complex

$$
\cdots \longrightarrow U_{-3 / 2} \stackrel{\bar{Q}}{\longrightarrow} U_{-1 / 2} \stackrel{\bar{Q}}{\longrightarrow} U_{1 / 2} \stackrel{\bar{Q}}{\longrightarrow} U_{3 / 2} \longrightarrow \cdots,
$$

where the $U_{\bar{q}}$ are states with definite $\bar{q}$ charge.

\footnotetext{
${ }^{3}$ The $\mathrm{U}(1)$ factor is precisely the left-moving R-symmetry.
} 


\subsection{Quantum Numbers in Twisted Sectors}

The remaining question to be addressed is the computation of the $E, q$ and $\bar{q}$ quantum numbers in the twisted sectors. The $E$ and $q$ quantum numbers of the twisted vacua can be obtained by using the UV $N=2$ algebra. In the $k$-th sector the fields have a twisted moding

$$
\begin{aligned}
x^{i}(z) & =\sum_{s \in \mathbb{Z}-\nu_{i}} x_{s}^{i} z^{-s-h_{i}}, & \gamma^{i}(z) & =\sum_{s \in \mathbb{Z}-\widetilde{\nu}_{i}} \gamma_{s}^{i} z^{-s-\widetilde{h}_{i}} \\
2 \partial \bar{x}^{i}(z) & =\sum_{s \in \mathbb{Z}+\nu_{i}} \rho_{s}^{i} z^{-s+h_{i}-1}, & \bar{\gamma}^{i}(z) & =\sum_{s \in \mathbb{Z}+\widetilde{\nu}_{i}} \bar{\gamma}_{s}^{i} z^{-s+\widetilde{h}_{i}-1},
\end{aligned}
$$

where $2 h_{i}=\alpha_{i}, 2 \widetilde{h}_{i}=1+\alpha_{i}$, and

$$
\begin{aligned}
\nu_{i} & =\frac{k \alpha_{i}}{2} \quad(\bmod 1) & 0 & \leq \nu_{i}<1 \\
\widetilde{\nu}_{i} & =\frac{k\left(\alpha_{i}-1\right)}{2}(\bmod 1) & -1 & <\widetilde{\nu}_{i} \leq 0 .
\end{aligned}
$$

The Fock vacuum $|k\rangle$ is defined as the state annihilated by all positive modes. The leftmoving quantum numbers of $|k\rangle$ can be computed by working out the one-point functions of $J$ and $T$ via the mode expansion. For instance, we easily find

$$
\begin{aligned}
2\left\langle k\left|x^{i}(w+\epsilon) \partial \bar{x}^{i}(w)\right| k\right\rangle & =\epsilon^{-1}+\left(\nu_{i}-h_{i}\right) w^{-1}+O(\epsilon), \\
\left\langle k\left|\gamma^{i}(w+\epsilon) \bar{\gamma}^{i}(w)\right| k\right\rangle & =\epsilon^{-1}+\left(1+\widetilde{\nu}_{i}-\widetilde{h}_{i}\right) w^{-1}+O(\epsilon) .
\end{aligned}
$$

Using a point-splitting regularization for $J$, these correlators imply

$$
w\langle k|J(w)| k\rangle=\sum_{i}\left[\left(\alpha_{i}-1\right)\left(1+\widetilde{\nu}_{i}-\widetilde{h}_{i}\right)-\alpha_{i}\left(\nu_{i}-h_{i}\right)\right],
$$

whence we read off the left-moving charge of $|k\rangle$ as

$$
q_{k}=\sum_{i}\left[\left(\alpha_{i}-1\right)\left(\widetilde{\nu}_{i}+\frac{1}{2}\right)-\alpha_{i}\left(\nu_{i}-\frac{1}{2}\right)\right] .
$$

Similarly, we obtain the weight via $w^{2}\langle k|T(w)| k\rangle=h_{k}$ :

$$
h_{k}=\frac{3}{8}+\Delta_{k}, \quad \Delta_{k}=\frac{1}{2} \sum_{i}\left[\nu_{i}\left(1-\nu_{i}\right)+\widetilde{\nu}_{i}\left(1+\widetilde{\nu}_{i}\right)\right] .
$$

To obtain the energy, we must transform from the plane to the cylinder and remember to include contributions from the other left-moving degrees of freedom. In sectors with $k$ even, we find $E_{k}=0$ - an answer in line with the $(2,2)$ supersymmetry. For $k$ odd, the result is

$$
E_{k}=\underbrace{\frac{3}{8}+\Delta_{k}-\frac{9}{24}}_{\text {LG }}+\underbrace{0-\frac{5}{24}}_{\mathrm{SO}(10)}+\underbrace{0-\frac{8}{24}}_{\mathrm{E}_{8}, \mathrm{NS}}+\underbrace{0-\frac{2}{24}}_{\text {space-time }}=-\frac{5}{8}+\Delta_{k} .
$$


The remaining quantum number is the right-moving R-charge. The simplest way to determine this is to note that in the UV the right-moving current $\bar{J}$ is related to the leftmoving current via $\bar{J}=J+J_{B}$, where $J_{B}$ assigns charge +1 to $\gamma^{i}$ and -1 to $\psi^{i}$. The $J_{B}$ symmetry is independent of $W$, and we can evaluate the charge of the twisted vacuum by simply setting $W=0$. Combining this with the result for $J$, we find

$$
\bar{q}_{k}=\sum_{i}\left[\alpha_{i}\left(\widetilde{\nu}_{i}+\frac{1}{2}\right)+\left(\alpha_{i}-1\right)\left(-\nu_{i}+\frac{1}{2}\right)\right] .
$$

Having determined the ground state quantum numbers, we will construct $E=q=0$ energy states by acting on $|k\rangle$ with the lowest excited modes, which we will denote by

$$
x_{i} \equiv x_{-\nu_{i}}^{i}, \quad \rho_{i} \equiv \rho_{\nu_{i}-1}^{i}, \quad \gamma_{i} \equiv \gamma_{-1-\widetilde{\nu}_{i}}^{i}, \quad \bar{\gamma}_{i} \equiv \bar{\gamma}_{\widetilde{\nu}_{i}}^{i} .
$$

As usual, an oscillator with mode $\nu$ will contribute $-\nu$ to the energy. In describing the $\bar{Q}$ cohomology we will also need the conjugate modes, which we will distinguish with a dagger:

$$
x_{i}^{\dagger} \equiv \rho_{\nu_{i}}^{i}, \quad \rho_{i}^{\dagger} \equiv x_{1-\nu_{i}}^{i}, \quad \gamma_{i}^{\dagger} \equiv \bar{\gamma}_{1+\widetilde{\nu}_{i}}^{i}, \quad \bar{\gamma}_{i}^{\dagger} \equiv \gamma_{-\widetilde{\nu}_{i}}^{i} .
$$

The $\bar{Q}$ operator takes the general form

$$
\bar{Q}=\sum_{i}\left\{\gamma_{i} \partial_{i} W_{1+\widetilde{\nu}_{i}}+\bar{\gamma}_{i}^{\dagger} \partial_{i} W_{\widetilde{\nu}_{i}}\right\} .
$$

\subsection{The Quintic}

We will now apply the method reviewed above to determine the singlet spectrum at the Landau-Ginzburg point in the moduli space of the quintic in $\mathbb{C P}^{4}$. This calculation was performed in [16], and we repeat it here to review the notation and also to remark on some universal features of such models. The theory is described by the superpotential $W\left(X_{0}, \ldots, X_{4}\right)$, a degree five polynomial. The R-charges of the fields are $\alpha_{i}=\frac{1}{5}$, so that the $\mathbb{Z}_{5}$ orbifold acts by multiplying the fields by fifth roots of unity. Thus, there are ten twisted sectors. CPT invariance allows us to restrict to $k=0, \ldots, 5$, and since we are interested in the singlets, we can restrict to odd $k$. The ground state quantum numbers and field modings for the sectors are given in table 2 .

Zero energy states with $q=0$ are only found in the $k=1$ and $k=3$ sectors. An explicit basis for these states is

$$
\begin{aligned}
& \bar{q}=-\frac{3}{2} \quad \bar{q}=-\frac{1}{2} \\
& \gamma_{i} \bar{\gamma}_{j}|1\rangle_{25} \longrightarrow F_{[4]}^{i} \gamma_{i}|1\rangle_{350} \\
& x_{i} \rho_{j}|1\rangle_{25}
\end{aligned}
$$

$$
x_{i} \bar{\gamma}_{j}|3\rangle_{25}
$$




\begin{tabular}{|c|c|c|c|c|c|}
\hline$k$ & $E_{k}$ & $q_{k}$ & $\bar{q}_{k}$ & $\nu$ & $\widetilde{\nu}$ \\
\hline 0 & 0 & $-\frac{3}{2}$ & $-\frac{3}{2}$ & 0 & 0 \\
\hline 1 & -1 & 0 & $-\frac{3}{2}$ & $\frac{1}{10}$ & $-\frac{2}{5}$ \\
\hline 2 & 0 & $\frac{3}{2}$ & $-\frac{3}{2}$ & $\frac{1}{5}$ & $-\frac{4}{5}$ \\
\hline 3 & $-\frac{1}{2}$ & -1 & $-\frac{1}{2}$ & $\frac{3}{10}$ & $-\frac{1}{5}$ \\
\hline 4 & 0 & $\frac{1}{2}$ & $-\frac{1}{2}$ & $\frac{2}{5}$ & $-\frac{3}{5}$ \\
\hline 5 & 0 & -2 & $\frac{1}{2}$ & $\frac{1}{2}$ & 0 \\
\hline
\end{tabular}

Table 2: Ground state quantum numbers and modings for the quintic.

Unless indicated otherwise, here and in what follows $F_{[d]}$ denotes a degree $d$ polynomial in the $x_{i}$ with respect to the multi-grading of the relevant homogeneous coordinate ring (in this case the $x_{i}$ just have charge 1 ). The subscript of the ket indicates the number of linearly independent states of each type. The map $\bar{Q}: U_{-3 / 2} \rightarrow U_{-1 / 2}$ is given by

$$
\bar{Q}=\bar{\gamma}_{i}^{\dagger} \partial_{i} W+\gamma_{i} \partial_{i j} W \rho_{j}^{\dagger}
$$

so that the image of an arbitrary state $|\psi\rangle \in U_{-3 / 2}$, specified by two matrices $c^{i j}$ and $d^{i j}$ is

$$
\bar{Q}|\psi\rangle=\bar{Q}\left\{4 c^{i j} \gamma_{i} \bar{\gamma}_{j}+d^{i j} x_{i} \rho_{j}\right\}|1\rangle=\left\{-4 c^{i j} \partial_{j} W+d^{k j} x_{k} \partial_{i j} W\right\} \gamma_{i}|1\rangle .
$$

What is the kernel of $\bar{Q}$ ? Since $W$ is quasi-homogeneous, $\operatorname{dim} \operatorname{ker} \bar{Q} \geq 1$ for any $W$. A $\bar{Q}$-closed state is obtained by setting $c^{i j}=d^{i j}=\delta^{i j}$. In fact, for generic $W$ this is the only $\bar{Q}$-closed state in $U_{-3 / 2}$. Its existence is not surprising: this is the vector multiplet corresponding to the unbroken $\mathrm{U}(1)_{L}$ symmetry. Thus, we find that there are generically 301 massless chiral singlets in the $k=1$ sector.

When $W$ is taken to be Fermat, we find that there are five $\bar{Q}$-closed states at $U_{-3 / 2}$, with $d^{i j}=d^{i} \delta^{i j}$ and $c^{i j}=d^{i j}$. Again, this is not a big surprise, since at this point in the moduli space the theory reduces to the Gepner model for $\oplus_{i=1}^{5} A_{4}^{i}$. This theory has five unbroken $\mathrm{U}(1)$ currents, each of which leads to a massless vector multiplet. So, at the Fermat point we find 305 massless chiral singlets at $k=1$.

The physics encoded by the change in $\operatorname{ker} \bar{Q}$ is just the supersymmetric Higgs mechanism: the disappearance of a massless vector multiplet is accompanied by the disappearance of a massless $\mathrm{E}_{6}$ singlet chiral multiplet. The number of massless vector multiplets is given by the number of decoupled components of the Landau-Ginzburg theory. For instance, turning on the unique monomial containing all the fields breaks all but one of the currents and leads to 301 massless singlets.

Finally, we turn to the $k=3$ sector. Here there are 25 zero energy states with $q=0$ as shown in 16 . Clearly $\bar{Q}=0$ in this sector, so that all of these states correspond to massless singlets. Combining these states with the $k=1$ singlets, we see that the theory contains 
326 massless singlets for generic $W$ and 330 at the Fermat point. Subtracting the $1+101$ $(2,2)$ moduli, we find 224 singlets that are not associated to extra $\mathrm{U}(1)$ gauge symmetries.

The $(2,2)$ Landau-Ginzburg Lagrangian can be deformed to a $(0,2)$ theory by replacing $\partial_{i} W$ with arbitrary quartic polynomials $W^{i}$ in the component Lagrangian. Although the $G^{ \pm}$ generators of the left-moving SCA are no longer conserved, the $J$ and $T$ still generate a leftmoving $\mathrm{U}(1)_{\mathrm{L}} \times$ Virasoro algebra. The computation of the ground-state quantum numbers and $\bar{Q}$ cohomology are unchanged by these deformations.

\subsection{Universal Structure in LG Singlet Spectrum}

The spectrum of singlets we have seen in the quintic in fact exhibits a pattern that is somewhat universal and will be useful in connecting the Landau-Ginzburg results to calculations in the large radius phase. Consider a Landau-Ginzburg model with $N=5$ fields and arbitrary charges $0<\alpha_{i} \leq \frac{1}{2}$ such that $\sum_{i}\left(1-2 \alpha_{i}\right)=3$. We will consider the $k=1$ and $k=3$ sectors of any such model and find that some singlet states are universally present.

To simplify expressions, note from (6) we have

$$
\widetilde{\nu}_{i}= \begin{cases}\nu_{i}-\frac{1}{2}, & 0<\nu_{i} \leq \frac{1}{2} \\ \nu_{i}-\frac{3}{2}, & \frac{1}{2} \leq \nu_{i}<1\end{cases}
$$

We begin with the $k=1$ sector. Here

$$
0<\nu_{i}=\frac{1}{2} \alpha_{i} \leq \frac{1}{4} \quad \text { and } \quad-\frac{1}{2}<\widetilde{\nu}_{i}=\nu_{i}-\frac{1}{2} \leq-\frac{1}{4}
$$

Inserting these values we find $E_{1}=-1$ and $\left(q_{1}, \bar{q}_{1}=\left(0,-\frac{3}{2}\right)\right.$. These values show immediately that zero-energy states will be given by

$$
|\psi\rangle=\left(F(x, \bar{\gamma})+G^{i}(x, \bar{\gamma}) \rho_{i}+H^{i}(x, \bar{\gamma}) \gamma_{i}\right)|1\rangle
$$

Since $\nu_{i} \propto \alpha_{i}$ functions of $x$ can be classified by their degree, $f_{[p]}\left(\lambda^{\alpha_{i} d} x_{i}\right)=\lambda^{p} f(x)$, so that $p$ determines the charge and energy of $f(x)$. We also use the notation $n_{i}=\alpha_{i} d \in \mathbb{Z}$. A possible chiral field with $\alpha=\frac{1}{2}$ must here be distinguished, so we use the somewhat clumsy notation

$$
\begin{array}{rlrl}
\alpha_{I} & =\frac{1}{2} & I & =0, \ldots, n-1 ; \\
0<\alpha_{a}<\frac{1}{2} & a & =n, \ldots, 4 .
\end{array}
$$

Clearly, $0 \leq n \leq 1$; if $n=0$ the first row is vacuous.

Considering the first term in $(20)$, the zero energy condition shows that

$$
\begin{gathered}
F(x, \bar{\gamma})=F_{[2 d]}(x)+F_{\left[d+n_{i}\right]}^{i}(x) \bar{\gamma}_{i}+F_{\left[n_{i}+n_{j}\right]}^{i j}(x) \bar{\gamma}_{i} \bar{\gamma}_{j}+\cdots \\
+F_{\left[n_{1}+\ldots+n_{m}-(m-2) d\right]}^{i_{1} \ldots i_{m}} \bar{\gamma}_{i_{1}} \cdots \bar{\gamma}_{i_{m}}+\cdots
\end{gathered}
$$


The expansion extends so long as there are terms for which the degree of $F$ is positive. All of these yield states at $q=2$. The second term potentially yields two types of zero energy states

$$
\begin{aligned}
& G^{I}(x, \bar{\gamma})=G_{\left[n_{I}\right]}^{I}(x)+\widetilde{G}^{I} \bar{\gamma}_{I} \\
& G^{a}(x, \bar{\gamma})=G_{\left[n_{a}\right]}^{a}(x) .
\end{aligned}
$$

States of the first type have $(q, \bar{q})=\left(0,-\frac{3}{2}\right)$ and represent singlets in vector multiplets. The second term in the first line contributes $n$ states at $(q, \bar{q})=\left(0,-\frac{5}{2}\right)$. The third term also yields two types of zero energy states

$$
H^{i}(x, \bar{\gamma})=H_{\left[d-n_{i}\right]}^{i}(x)+H_{\left[n_{j}-n_{i}\right]}^{i j}(x) \bar{\gamma}_{j}
$$

The first of these contributes states at $(q, \bar{q})=\left(0,-\frac{1}{2}\right)$ (chiral matter) and the second at $\left(0,-\frac{3}{2}\right)$ (vector multiplets). This exhausts the possible singlet states at $k=1$. The action of $\bar{Q}$ is determined from 15

$$
\bar{Q}\left(\widetilde{G}^{I} \bar{\gamma}_{I} \rho_{I}\right)=\widetilde{G}^{I} \partial_{I} W \rho_{I}, \quad \bar{Q}\left(G^{i} \rho_{i}\right)=\sum_{k} G^{i} \partial_{i k} W \gamma_{k}, \quad \bar{Q}\left(H^{i j} \bar{\gamma}_{j} \gamma_{i}\right)=H^{i j} \partial_{j} W \gamma_{i}
$$

We now move to the $k=3$ sector. In general we will not enumerate all possible states at $q=E=0$ or the action of $\bar{Q}$. Rather, we will show that certain states arise universally at $\bar{q}=-\frac{1}{2}$. Here a different distinction among the fields by weight is appropriate

$$
\begin{aligned}
& \frac{1}{3}<\alpha_{A} \leq \frac{1}{2} \quad \frac{1}{2}<\nu_{A} \leq \frac{3}{4} \quad-1<\widetilde{\nu}_{A} \leq-\frac{3}{4} \quad A=0, \ldots, m-1 \text {; } \\
& 0<\alpha_{a} \leq \frac{1}{3} \quad 0<\nu_{a} \leq \frac{1}{2} \quad-\frac{1}{2}<\widetilde{\nu}_{a} \leq 0 \quad a=m, \ldots, 4 .
\end{aligned}
$$

Clearly $n \leq m \leq 2$. Inserting these values we find that the ground state is characterized by

$$
\begin{aligned}
2 E_{3} & =m-1-3 \sum_{A} \alpha_{A} ; \\
\left(q_{3}, \bar{q}_{3}\right) & =\left(m-1-\sum_{A} \alpha_{A},-\frac{1}{2}-\sum \alpha_{A}\right) .
\end{aligned}
$$

We will not attempt a complete characterization of all zero-energy states in this sector but note that the following states always arise:

$$
K_{\left[n_{A}\right]}^{A} \prod_{B \neq A} \gamma_{B}|3\rangle, \quad K_{\left[n_{a}\right]}^{a}(x) \bar{\gamma}_{a} \prod_{A} \gamma_{A}|3\rangle
$$

Note that since we still have $\nu_{i} \propto \alpha_{i}$ we classify functions of $x$ by degree as above. All of these states have $(q, \bar{q})=\left(0,-\frac{1}{2}\right)$ and represent scalars in chiral multiplets. The first term obviously contributes when $m$ is nonzero. The coefficient space for these states is identical to the first row of $\bar{q}=-\frac{3}{2}$ states in the $k=1$ sector in table 3 and in terms of counting 


$$
\begin{aligned}
& \bar{q}=-\frac{5}{2} \quad \bar{q}=-\frac{3}{2} \quad \bar{q}=-\frac{1}{2} \\
& \widetilde{G}_{[0]}^{I} \bar{\gamma}_{I} \rho_{I}|1\rangle \stackrel{\bar{Q}}{\longrightarrow} \underset{\oplus_{n_{j} \geq n_{i}} H_{\left[n_{j}-n_{i}\right]}^{i j} \bar{\gamma}_{j} \gamma_{i}|1\rangle}{\oplus} \stackrel{\oplus_{i} G_{\left[n_{i}\right]}^{i} \rho_{i}|1\rangle}{\bigoplus} \stackrel{\bar{Q}}{\longrightarrow} \oplus_{k} H_{\left[d-n_{k}\right]}^{k} \gamma_{k}|1\rangle \\
& \oplus_{A} K_{\left[n_{A}\right]}^{A} \prod_{B \neq A} \gamma_{B}|3\rangle \\
& \oplus_{a} K_{\left[n_{a}\right]}^{a} \bar{\gamma}_{a} \prod_{A} \gamma_{A}|3\rangle
\end{aligned}
$$

Table 3: Universal Singlets in Landau-Ginzburg models.

singlets they explicitly "cancel them out." In general there can be other zero energy states at $k=3$. These can include states with charge $\left(0,-\frac{3}{2}\right)$ and nontrivial $\bar{Q}$ action, so not all of the states listed above are physical. In all cases we have observed, the coefficient spaces of these $\bar{q}=-\frac{3}{2}$ states are then repeated as coefficients of $\bar{q}=-\frac{1}{2}$ states in other sectors, effectively canceling again. We term this reappearance of $k=1$ states in higher sectors the "cascade." We will see an example of this in Section 5.2.3.

The set of states we have listed here is present in any Landau-Ginzburg model where the orbifold simply projects onto integral R-charges. In the case of the quintic this is the complete complement of singlets. In other models there will be additional states in various sectors, but these universally present states will figure in comparing the Landau-Ginzburg phase with the orbifold.

\subsection{More LG Orbifolds}

The methods described above are easily extended to study quotients of the Landau-Ginzburg models discussed above by further discrete symmetries. These will be useful when constructing the mirrors as Landau-Ginzburg models ${ }^{4}$ A model with charges $\alpha_{i}=n_{i} / d$ has a natural non- $R$ action of $\mathrm{U}(1)^{N}$ acting by phases on the worldsheet chiral multiplets $\Phi_{i}$. This is broken by the superpotential $W$, and for generic $W$ it is broken to $\mathbb{Z}_{d}$, by which we took the quotient above. Special nonsingular superpotentials leave a larger discrete subgroup $G \subset \mathrm{U}(1)^{N}$ unbroken, and we can construct quotient theories following the same steps as above, with the simplifying feature that the new symmetries act non-chirally. In what follows we will take $G$ to be abelian, and we will not consider general choices of discrete torsion in Landau-Ginzburg orbifolds [23].

The symmetry groups by which we quotient will act by phases on the chiral multiplets. We represent the group elements by $N$-tuples of rational numbers defined up to integers, so that the vector $w$ represents the action of $g \in G$ :

$$
g: \Phi_{i} \mapsto e^{2 \pi i w_{i}} \Phi_{i}
$$

\footnotetext{
${ }^{4}$ In addition, a generic Landau-Ginzburg phase of a GLSM will have such further quotients as part of the discrete gauge symmetry.
} 
generating a cyclic action of order $d(w)=\operatorname{gcd}\left(w_{i}\right)$.

To construct the quotient by a group generated by vectors $w^{(a)}$ we introduce twisted sectors labeled by $0 \leq t^{(a)} \leq d\left(w^{(a)}\right)-1$. The new symmetry is not an $R$-symmetry so the twists of bosons and fermions (6) are modified to

$$
\begin{aligned}
& \nu_{i}=\frac{k \alpha_{i}}{2}+\sum_{a} t^{(a)} w_{i}^{(a)}(\bmod 1) \quad 0 \leq \nu_{i}<1 \\
& \widetilde{\nu}_{i}=\frac{k\left(\alpha_{i}-1\right)}{2}+\sum_{a} t^{(a)} w_{i}^{(a)}(\bmod 1) \quad-1<\widetilde{\nu}_{i} \leq 0 .
\end{aligned}
$$

We also introduce a projection onto states invariant under (29).

The $G$ action in twisted sectors is determined by the requirement of modular invariance from (29). For a non-chiral symmetry this is the same as the action in the untwisted sector, and the twisted vacua are uncharged. The GSO projection, however, is chiral and thus sectors with nonzero $k$ will in general carry a $G$-charge $5^{5}$ The charge is easily computed by working with the UV fields and free OPEs. Since the twisted bosons make no contribution, it is sufficient to consider the action of $g$ on the fermions, which we express as a subgroup of the $\mathrm{U}(1)$ vectorial symmetry with current $J_{G}=\sum_{i} w_{i}\left(\gamma^{i} \bar{\gamma}^{i}+\psi^{i} \bar{\psi}^{i}\right)$. The full $\mathrm{U}(1)$ symmetry is broken by the superpotential couplings, but since $W$ is $G$-invariant, we can use this embedding to compute the $G$-charge. The result is that a twisted vacuum $\left|k, t^{(a)}\right\rangle$ transforms by a phase $e^{2 \pi i q_{g}}$, with

$$
q_{g}=\sum_{i} w_{i}\left(\widetilde{\nu}_{i}-\nu_{i}+1\right) \quad(\bmod 1) .
$$

The quotients of interest to us will be those preserving space-time supersymmetry. This requires that the left-moving spectral flow operator, in the $k=1$ sector, be preserved by the projection. We can construct this operator in the free field representation of section 2.1 and find that it is preserved if

$$
\sum_{i} w_{i}=0 .
$$

Note that since 29 depends on $w_{i}$ only modulo integers, this condition is equivalent to the more familiar

$$
\sum_{i} w_{i} \in \mathbb{Z}
$$

in terms of restricting the allowable quotients. In the twisted sectors, (31) will hold when $w$ are chosen to satisfy the more stringent condition.

\subsection{Gepner Models and Mirror Symmetry}

For special values of the superpotential couplings, the Landau-Ginzburg model in all of our examples is an exactly solvable theory [24]. Prior to the orbifold of section 2.2 we have a

\footnotetext{
${ }^{5}$ We would like to thank B. Wurm for his help in clarifying this point.
} 
product of $(2,2)$ minimal models. This, of course, allows a calculation of the spectrum of singlets at this point in the moduli space, but this is equivalent to a special case of the Landau-Ginzburg calculation, as discussed above. The utility, to our work, of the Gepner model, is that at this point we have a construction [25] of the mirror model as an orbifold, as well as an explicit mirror map in terms of the Gepner construction. This allows us to find the singlet states in the mirror model corresponding to the states we enumerate using the Landau-Ginzburg construction. We can then construct the mirror as a Landau-Ginzburg orbifold and study the dependence of the singlet spectrum on the mirror superpotential. Since deformations of the mirror superpotential are mapped to Kähler deformations in the original model, we can thus predict which singlet states will be lifted by Kähler deformations away from the Landau-Ginzburg locus.

In all of our examples, the exactly solvable superpotential will be a sum of terms of the form $x_{i}^{k_{i}+2}$, corresponding to an $A_{k_{i}+1}$ minimal model at level $k_{i}$ and of the form $x_{i}^{l_{i}+1}+x_{i} y_{i}^{2}$, corresponding to a $D_{l_{i}+2}$ minimal model at even level $k_{i}=2 l_{i}$, leading to a tensor product of $n$ minimal models $\sqrt[6]{6}$ Primary fields in the level- $k$ minimal model are labeled $\Phi_{q, s ; \bar{q}, \bar{s}}^{l, \bar{l}}$ where $0 \leq l, \bar{l} \leq k$, subject to the identifications

$$
q \sim q+2(k+2) \quad s \sim s+4,
$$

(and the same for $\bar{q}, \bar{s}$ ) as well as

$$
\Phi_{q, ; ; \bar{q}, \bar{s}}^{l, \bar{l}} \sim \Phi_{q+k+2, s+2 ; \bar{q}+k+2, \bar{s}+2}^{k-l, k \bar{l}} .
$$

Fields with even (odd) $s$ create states in the NS (R) sector.7 We use Gepner's notation $\left(\begin{array}{c}l \\ q\end{array}\right) \mid\left(\begin{array}{c}\bar{l} \\ \bar{q}\end{array}\right)$ for the state created by $(35)$. The $R$-charge and conformal weight of a state are given by

$$
\begin{aligned}
& q=-\sum_{i} \frac{\tilde{q}_{i}}{k_{i}+2}+\frac{s_{i}}{2} \\
& h=\sum_{i} \frac{l_{i}\left(l_{i}+2\right)-q_{i}^{2}}{4 k_{i}+8}+\frac{s_{i}^{2}}{8},
\end{aligned}
$$

for $q_{i}, s_{i}$ in the standard range $\left|q_{i}-s_{i}\right| \leq l,-1 \leq s_{i} \leq 2$, where $\tilde{q}_{i}=q_{i}+s_{i}$ for $\mathrm{R}$ states and $\tilde{q}_{i}=q_{i}$ for NS states.

The minimal model at level $k$ has a partition function

$$
Z=\frac{1}{2} \sum_{l+q+s \in 2 \mathbb{Z}} A_{l, \bar{l}} \chi_{q, s}^{l} \chi_{q, s}^{\bar{l} *}
$$

\footnotetext{
${ }^{6}$ We hope there will be no confusion between the minimal model levels labeled by $k_{i}$ and the twisted sectors labeled by $k$.

${ }^{7}$ In fact, $s=2$ states are not primary but after the orbifold they do create highest weight states; this is related to the fact that the quotient projects out some modes of the supercurrents in the individual minimal models.
} 
where $A_{l, \bar{l}}$ is the appropriate affine modular invariant at level $k+2$ and the factor of $\frac{1}{2}$ reflects the identification (35). The model enjoys a discrete symmetry $G_{k}=\mathbb{Z}_{k+2} \times \mathbb{Z}_{2}$ under which the state $\left(\begin{array}{c}l \\ q s\end{array}\right)$ has weights $q$, s. In the associated Landau-Ginzburg model we will be interested in the $\mathbb{Z}_{k+2}$ subgroup of this generated by $x_{i} \mapsto e^{2 \pi i / k_{i}+2} x_{i}$ in the $A_{k_{i}+1}$ model and by $x_{i} \mapsto e^{2 \pi i / l_{i}+1} x_{i}$ and $y_{i} \mapsto-y_{i}$ in the $D_{l_{i}+2}$ model.

The Gepner construction of a string vacuum as a quotient of the tensor product was introduced in Section 2.2. We add free fields and perform a quotient by $\mathbb{Z}_{d} \times \mathbb{Z}_{2}^{n}$. The quotient introduces twisted sectors in which $\bar{q}_{i}, \bar{s}_{i}$ differ from $q_{i}, s_{i}$ by $k$ and additional twists in which any two $\bar{s}$ indices are shifted by 2 . The gauge symmetry of the model is $\mathrm{E}_{6} \times \mathrm{E}_{8} \times \mathrm{U}(1)^{n-1}$. The $\mathrm{SO}(10)$-neutral scalars in chiral multiplets are states with $\bar{q}=1, \bar{h}=\frac{1}{2}$ and $q=h=0$. The corresponding fermion states are obtained by applying the spacetime supersymmetry generator, shifting $\bar{q}, \bar{s}$ by one, and leading to $\bar{q}=-\frac{1}{2}$. We will denote states in the resulting model by

$$
\left(\begin{array}{cc}
l_{1} \\
q_{1} s_{1}
\end{array}\right) \cdots\left(\begin{array}{c}
l_{n} \\
q_{n} s_{n}
\end{array}\right) \mid\left(\begin{array}{c}
\bar{l}_{1} \\
\bar{q}_{1} \bar{s}_{1}
\end{array}\right) \cdots\left(\begin{array}{c}
\bar{l}_{n} \\
\bar{q}_{n} \bar{s}_{n}
\end{array}\right) .
$$

The mirror model is constructed as a further quotient by a subgroup of $G$, essentially the subgroup under which the spacetime supercharge is invariant. The quotient introduces twisted sectors in which $\bar{q}_{j}$ is shifted relative to $q_{j}$ by $2 t_{a} m^{(a)}$ for a lattice generated by a set of integer vectors $m^{(a)}$ (and the associated projection). The result of the construction is 25 a model in which the primary fields are related to those of the original theory by $q \mapsto-q, s \mapsto-s$. The orbifold construction can be realized in the Landau-Ginzburg model as a quotient following Section 2.6 with the action on the chiral superfields given by

$$
\begin{array}{cc}
w_{j}^{(a)}=\frac{m_{j}^{(a)}}{k_{j}+2} & \text { for } A \text {-type } x_{j}^{k_{j}+2} \\
w_{j, x}^{(a)}=\frac{2 m_{j}^{(a)}}{k_{j}+2}, \quad w_{j, y}^{(a)}=\frac{m_{j}^{(a)}}{2} & \text { for } D \text {-type } x_{j}^{\left(k_{j}+2\right) / 2}+x_{j} y_{j}^{2}
\end{array}
$$

To use mirror symmetry to study the behavior of the singlet spectrum under Kähler deformations away from the Gepner point, we first find the spectrum of singlets in the original model. For each of these we construct the mirror state and identify the twisted sector in the mirror quotient in which it arises. We then consider the mirror Landau-Ginzburg model constructed as an orbifold. The mirror superpotential will admit polynomial deformations related by the monomial-divisor mirror map to the toric Kähler deformations in the original model. In the relevant twisted sectors, we can study the change in $\bar{Q}$ cohomology when the superpotential is deformed, thus identifying which singlet states are lifted under Kähler deformations away from the Gepner point. 


\section{Deformations of the Tangent Bundle}

\subsection{The tangent sheaf}

Let $T$ denote the tangent sheaf (or bundle - we will use the terms interchangeably here) of a Calabi-Yau threefold $X$. We are interested in first order deformations of $T$ since they correspond classically to massless fields allowing, to first order, a deformation of a $(2,2)$ model to a $(0,2)$-model.

Such deformations are given by $\operatorname{Ext}_{X}^{1}(T, T)=H^{1}(X, \operatorname{End}(T))$. For a simple argument we refer to [26], chapter 15. Methods of computing $H^{1}(\operatorname{End}(T))$ have been studied for some time [5, 27, 28] for cases of Calabi-Yau manifolds in products of projective spaces. Here we give a method that is reasonably direct for complete intersections in toric varieties.

Let $V$ be a compact toric variety and let $X$ be a Calabi-Yau complete intersection within $V$. That is, we are in the context of Calabi-Yau's as studied in 29, 30. We quickly review the construction of $V$ to fix notation. Let $x_{0}, \ldots, x_{N-1}$ be the homogeneous coordinates on $V$. That is, we have a homogeneous coordinate ring in the sense of Cox [31]

$$
R=\mathbb{C}\left[x_{0}, \ldots, x_{N-1}\right] .
$$

We now have a short exact sequence

$$
0 \longrightarrow M \longrightarrow \mathbb{Z}^{\oplus N} \stackrel{\Phi}{\longrightarrow} D \longrightarrow 0,
$$

where $D$ is a lattic $\left.\right|^{8}$ of rank $r$. Each column of the matrix $\Phi$ can be thought of as a $\mathrm{U}(1)^{r}$ charge vector of the coordinates $x_{i}$. That is, $R$ has the structure of an $r$-multigraded ring.

The toric variety is given as

$$
V=\frac{\operatorname{Spec}(R)-Z(B)}{\left(\mathbb{C}^{*}\right)^{r}},
$$

where $B$ is the "irrelevant ideal" in $R$ and $Z(B)$ is the associated subvariety of $\mathbb{C}^{N} . B$ is determined combinatorially from the fan describing $V$.

Let $\mathbf{v}$ denote an element of the lattice $D$, i.e., an $r$-vector. If $M$ is a multigraded $R$ module then we may shift multi-gradings to form $M(\mathbf{v})$ in the usual way. Correspondingly, if $\mathscr{O}_{V}$ is the structure sheaf of $V$, then we may denote by $\mathscr{O}_{V}(\mathbf{v})$ the twisted sheaf associated to the module $R(\mathbf{v})$. Line bundles on $V$ correspond to $\mathscr{O}_{V}(\mathbf{v})$ for various $\mathbf{v} \in D$. If $V$ is smooth then every element of $D$ defines a line bundle.

Let $\mathbf{q}_{i}$ denote the row vectors of the transpose of $\Phi$. That is, $\mathbf{q}_{i}$ represents the multigrading of the homogeneous coordinate $x_{i}$. Let $T_{V}$ be the tangent sheaf of $V$. Assuming $V$ is smooth, we have the generalization of the Euler exact sequence for a toric variety 32

$$
0 \longrightarrow \mathscr{O}_{V}^{\oplus r} \stackrel{x_{i} \mathbf{q}_{i}}{\longrightarrow} \bigoplus_{i=0}^{N-1} \mathscr{O}_{V}\left(\mathbf{q}_{i}\right) \longrightarrow T_{V} \longrightarrow 0
$$

\footnotetext{
${ }^{8}$ Assumed to be torsion-free.
} 
Let

$$
\mathbf{Q}=\sum_{i=0}^{N-1} \mathbf{q}_{i}
$$

Suppose $X$ is a smooth hypersurface in $V$ representing the anticanonical class. Then $X$ is a Calabi-Yau manifold and we have the adjunction exact sequence:

$$
0 \longrightarrow T_{X} \longrightarrow T_{V \mid X} \longrightarrow \mathscr{O}_{X}(\mathbf{Q}) \longrightarrow 0,
$$

where we denote a restriction of $\mathscr{O}_{V}(\mathbf{v})$ to $X$ by $\mathscr{O}_{X}(\mathbf{v})$.

Since all the sheaves in (42) are locally-free, we may restrict to $X$ and the sequence will remain exact. Combining this with the sequence (44) yields the following fact. The complex

$$
0 \longrightarrow \mathscr{O}_{X}^{\oplus r} \stackrel{x_{i} \mathbf{q}_{i}}{\longrightarrow} \bigoplus_{i} \mathscr{O}_{X}\left(\mathbf{q}_{i}\right) \stackrel{\partial_{i} W}{\longrightarrow} \mathscr{O}_{X}(\mathbf{Q}) \longrightarrow 0
$$

is exact everywhere except the middle term where the cohomology is isomorphic to the tangent sheaf $T_{X}$. Here $W$ denotes the defining equation for the hypersurface $X$.

It is easy to generalize this to the case of a complete intersection. Suppose $X$ is defined by an intersection of $W_{1}=W_{2}=\ldots=0$. Let each $W_{a}$ have grade $\mathbf{Q}_{a}$. Then the tangent sheaf is given by the cohomology of

$$
0 \longrightarrow \mathscr{O}_{X}^{\oplus r} \stackrel{x_{i} \mathbf{q}_{i}}{\longrightarrow} \bigoplus_{i} \mathscr{O}_{X}\left(\mathbf{q}_{i}\right) \stackrel{\partial_{i} W_{a}}{\longrightarrow} \bigoplus_{a} \mathscr{O}_{X}\left(\mathbf{Q}_{a}\right) \longrightarrow 0
$$

For the remainder of the paper we assume $X$ is a hypersurface.

Before heading into the more complicated $H^{1}\left(\operatorname{End}\left(T_{X}\right)\right)$ computation it will be useful to consider the cohomology of the tangent sheaf itself. It is most convenient to use the language of the derived category $\mathbf{D}(X)$ to manipulate the tangent sheaf. The tangent sheaf is equivalent in $\mathbf{D}(X)$ to the complex 45, where the middle position of 45 is considered position zero. Suppose we have an object $\mathscr{E} \bullet$ in $\mathbf{D}(X)$ represented by a complex

$$
\cdots \longrightarrow \mathscr{E}^{-1} \longrightarrow \mathscr{E}^{0} \longrightarrow \mathscr{E}^{1} \longrightarrow \cdots
$$

We can consider the total cohomology (or hypercohomology) of this complex $H^{n}\left(\mathscr{E}^{\bullet}\right)$. There is a spectral sequence with 33

$$
E_{1}^{p, q}=H^{q}\left(\mathscr{E}^{p}\right),
$$

which converges to $H^{p+q}(\mathscr{E} \bullet)$. This gives a method of computing the cohomology of the 
tangent sheaf. The $E_{1}$ stage of the spectral sequence is

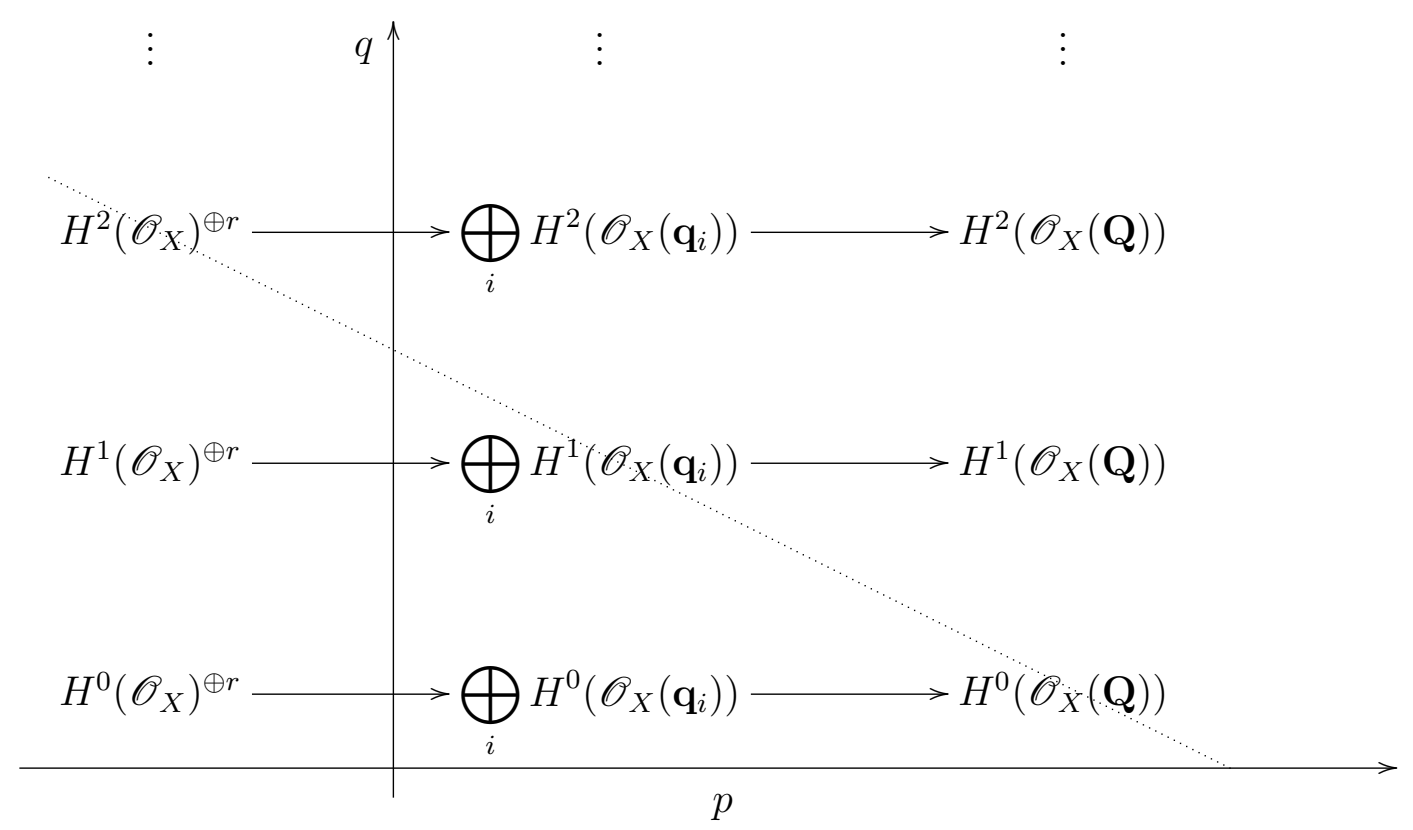

Fortunately it is straight-forward to compute the cohomology groups $H^{n}\left(V, \mathscr{O}_{X}(\mathbf{v})\right)=$ $H^{n}\left(X, \mathscr{O}_{X}(\mathbf{v})\right)$. First, the cohomology groups $H^{n}\left(V, \mathscr{O}_{V}(\mathbf{v})\right)$ can be computed. Actually we need to study these cohomology groups in detail and we give a relevant method (if not the most efficient) in the appendix. Then one may use exact sequences of the form

$$
0 \longrightarrow \mathscr{O}_{V}(\mathbf{v}-\mathbf{Q}) \longrightarrow \mathscr{O}_{V}(\mathbf{v}) \longrightarrow \mathscr{O}_{X}(\mathbf{v}) \longrightarrow 0
$$

to restrict to $X 9$

The dotted line in the spectral sequence (49) represented terms which contribute to $H^{1}\left(T_{X}\right)$, that is, deformations of complex structure. Since $X$ is a Calabi-Yau threefold we know that $H^{1}\left(\mathscr{O}_{X}\right)=H^{2}\left(\mathscr{O}_{X}\right)=0$. Also, since $\mathbf{Q}$ corresponds to an ample divisor, $H^{1}\left(\mathscr{O}_{X}(\mathbf{Q})\right)=0$. The contribution to $H^{1}\left(T_{X}\right)$ from the zeroth row (i.e., $q=0$ ) corresponds to the cokernel of the $d_{1}$ map induced from the complex 45). This is given by elements of $H^{0}\left(\mathscr{O}_{X}(\mathbf{Q})\right)$ which are not multiples of $\partial W / \partial x_{i}$. This is immediately recognizable as deformations of the defining polynomial $W$ modulo reparametrizations. These are the usual "polynomial deformations" of $X$. The spectral sequence then yields the following little result:

Theorem 1 The non-polynomial deformations of complex structure for a Calabi-Yau hypersurface $X$ in a toric variety are given by

$$
\bigoplus_{i} H^{1}\left(\mathscr{O}_{X}\left(\mathbf{q}_{i}\right)\right)
$$

\footnotetext{
${ }^{9}$ Perhaps the easiest way to compute this is to express it in terms of a module as a cokernel as in 50 and then compute the cohomology using the Macaulay 2 package 34 .
} 
This should be compared with a similar result obtained for deformations of complex structure of smooth projective toric varieties 35$]$.

\section{$3.2 \operatorname{End}(T)$}

We would now like to compute the cohomology groups $H^{k}(X, \operatorname{End}(T))$. These groups may also be written $\operatorname{Ext}_{X}^{1}(T, T)$. The machinery of the derived category is well-suited to compute these cohomology groups as we discuss. We refer to [36, 37] for more details.

Given a complex

$$
\mathscr{E} \bullet=\cdots \longrightarrow \mathscr{E}^{-1} \longrightarrow \mathscr{E}^{0} \longrightarrow \mathscr{E}^{1} \longrightarrow \cdots,
$$

of coherent sheaves and a similar complex $\mathscr{F}^{\bullet}$ we may form an object in $\mathbf{D}(X)$ which represents the object $\mathscr{H} o m(\mathscr{E}, \mathscr{F})$. It is given by the complex whose $n$th term is

$$
\mathscr{H} o m\left(\mathscr{E} \bullet, \mathscr{F}^{\bullet}\right)^{n}=\bigoplus_{j=i+n} \mathscr{H}_{0 m}\left(\mathscr{E}^{i}, \mathscr{F}^{j}\right)
$$

If $\phi \in \mathscr{H} o m\left(\mathscr{E} \bullet, \mathscr{F}^{\bullet}\right)^{n}$ then we define the differential of this new complex by

$$
d^{n}(\phi)=d_{\mathscr{F}} \phi-(-1)^{n} \phi d_{\mathscr{E}},
$$

as in [38]. The total cohomology of the object $\mathscr{H}$ om $(\mathscr{E}, \mathscr{F})$ represents the "hyperext" groups $\operatorname{Ext}(\mathscr{E}, \mathscr{F})$.

Let us apply the above to the case of the tangent sheaf. From (45) we have a complex representing $\mathscr{H} o m(T, T)$ given by

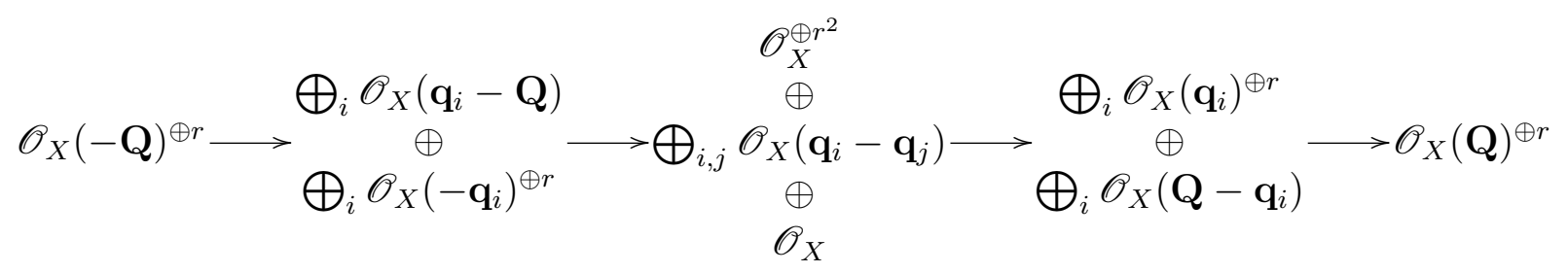

where the dotted line represents position zero. The maps in this complex are derived from $x_{i} \mathbf{q}_{i}$ and $\partial_{i} W$ by using (54). This yields

Theorem 2 There is a spectral sequence whose $E_{1}$ term is given by

$$
E_{1}^{p, q}=H^{q}\left(X, \mathscr{H} o m(T, T)^{p}\right),
$$

where $\mathscr{H} o m(T, T)$ is given by (55). This converges to

$$
H^{n}(X, \operatorname{End}(T)) \cong \bigoplus_{p+q=n} E_{\infty}^{p, q}
$$

This theorem gives a practical method of computing $H^{1}(\operatorname{End}(T))$ as we discuss in several examples below. 


\subsection{The Quintic}

The quintic threefold in $\mathbb{P}^{4}$ provides a simple example to demonstrate theorem 2, As we will see in this paper, the quintic is deceptively simple and fails to demonstrate most of the interesting phenomena that can happen for counting singlets. Nevertheless it always provides a good example to start with.

For the quintic, the tangent complex 45) becomes

$$
\mathscr{O}_{X} \stackrel{x_{i}}{\longrightarrow} \mathscr{O}_{X}(1)^{\oplus 5} \stackrel{\partial_{i} W}{\longrightarrow} \mathscr{O}_{X}(5) \text {. }
$$

Before writing down the full spectral sequence we should note that Serre duality gives

$$
H^{k}\left(X, \mathscr{O}_{X}(\mathbf{v}) \cong H^{3-k}\left(X, \mathscr{O}_{X}(-\mathbf{v})\right) .\right.
$$

This makes the second and third row of the spectral sequence copies of row one and zero written in reverse. We obtain

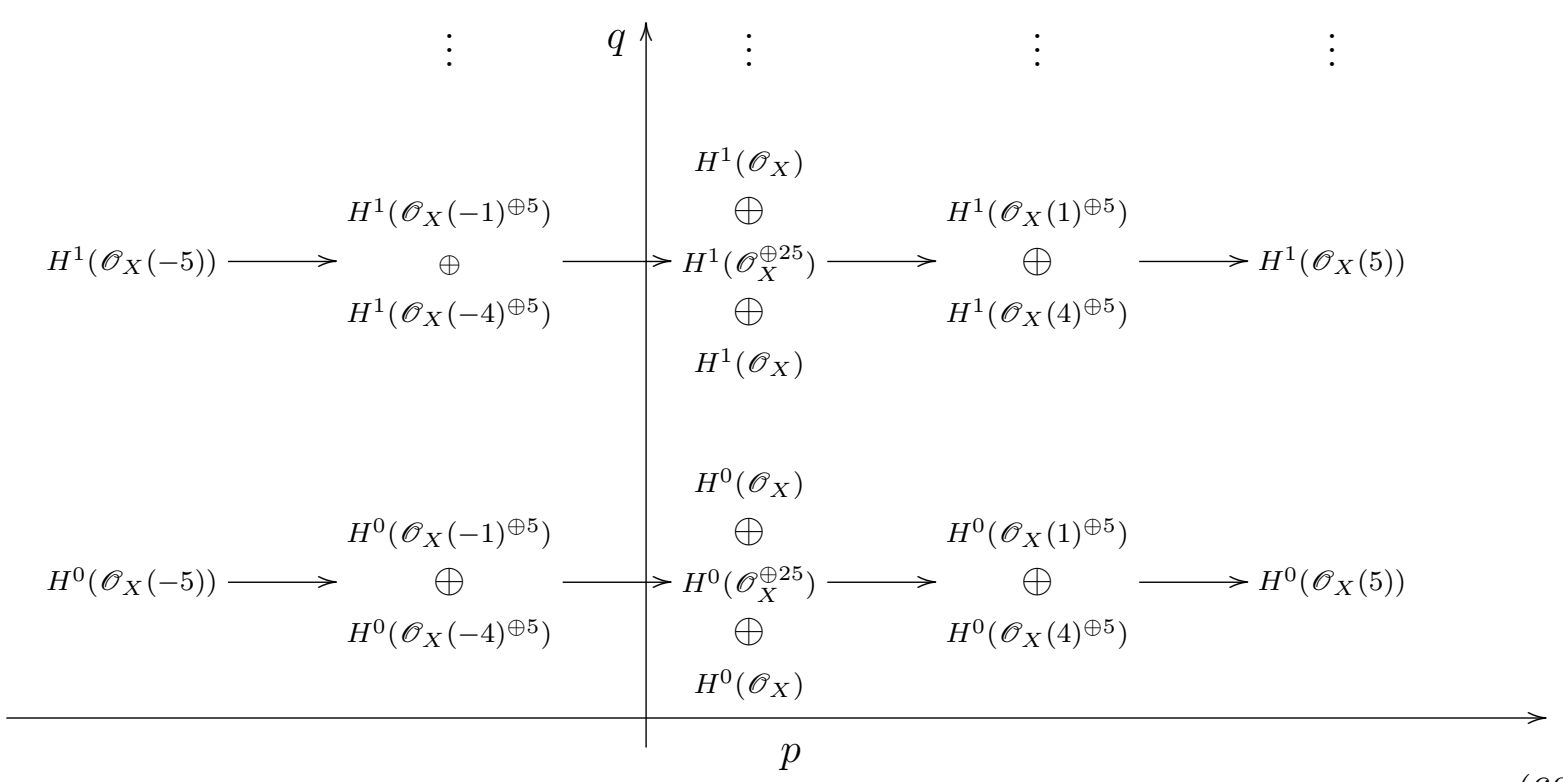

That is,

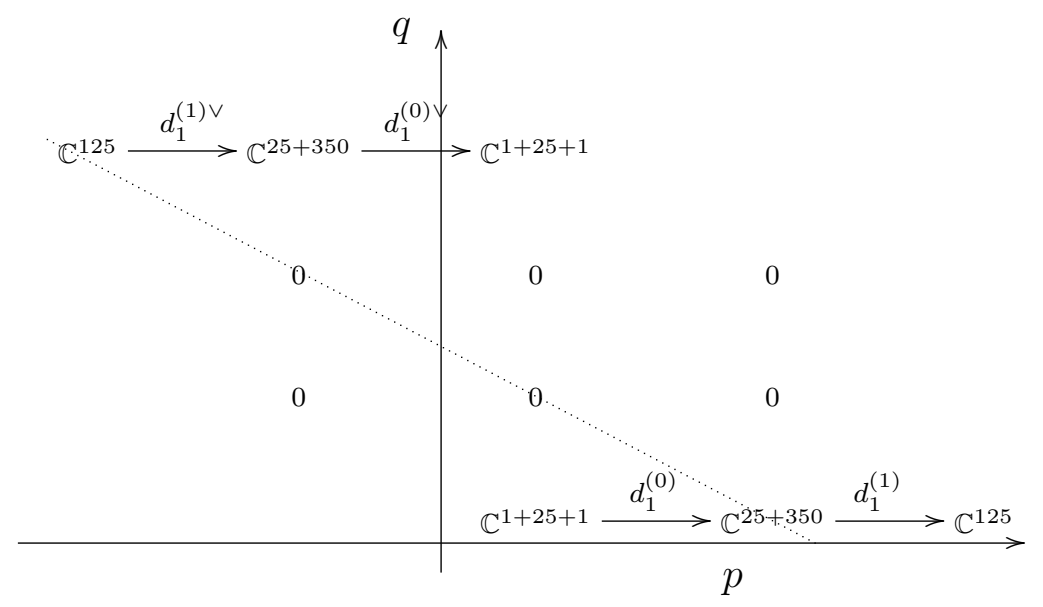


where we show contributions to $H^{1}(\operatorname{End}(T))$ with the dotted line.

To compute the $E_{2}$ stage of the spectral sequence requires an explicit determination of the $d_{1}$ maps in (61). This is not so bad since the monomials of degree $n$ form a basis (up to the quintic defining equation) of $H^{0}(\mathscr{O}(n))$. Actually, since the holonomy of the quintic threefold is precisely $\mathrm{SU}(3)$, the tangent sheaf is irreducible and so, by Schur's lemma, $H^{0}(\operatorname{End}(T))$ has dimension one. This means the map $d_{1}^{(0)}$ in 61 has a one-dimensional kernel.

The $\operatorname{map} d_{1}^{(1)}$ is of the form

$$
\begin{aligned}
& H^{0}\left(\mathscr{O}_{X}(1)\right)^{\oplus 5} \\
& H^{0}\left(\mathscr{O}_{X}(4)\right)^{\oplus 5}
\end{aligned}
$$

Since clearly any degree 5 polynomial can be expressed as a sum $\sum_{i} x_{i} g_{i}$, for quartic $g_{i}$ 's, the $\operatorname{map} d_{1}^{(1)}$ is surjective for any $W$.

Thus the spectral sequence degenerates at $E_{2}$ and we obtain

$$
\begin{aligned}
\operatorname{dim} H^{1}(X, \operatorname{End}(T)) & =25+350-125-1-25 \\
& =224,
\end{aligned}
$$

in agreement with known results [39] and section 2.4. Moreover, all 224 singlets correspond to $(0,2)$ GLSM deformations.

\section{Relating the Computations}

The main point of this work lies in comparing calculations of the singlet spectrum valid at various loci in the moduli space of $(2,2)$ theories. At different loci we apply different techniques, and in comparing the results we can find interesting relations between the calculations.

\section{1 $\operatorname{End}(T)$ and GLSM Deformations}

The spectral sequence of the section 3.2 is closely related to the $(0,2)$ GLSM holomorphic parameters studied in [9]. In addition to the toric Kähler parameters, the $(0,2)$ superpotential is encoded by the maps in the complex

$$
0 \longrightarrow \mathscr{O}_{V}^{\oplus r} \stackrel{\left(\begin{array}{l}
\mathbf{E}_{0} \\
\mathbf{E}_{i}
\end{array}\right)}{\longrightarrow} \mathscr{O}_{V} \stackrel{\oplus}{\mathscr{O}_{i}} \stackrel{\left(W, J_{i}\right)}{\longrightarrow} \mathscr{O}_{V}(\mathbf{Q}) \longrightarrow 0,
$$

where $W \in H^{0}\left(\mathscr{O}_{V}(\mathbf{Q})\right)$ specifies the hypersurface. In order for this to be a complex, this data must satisfy

$$
\sum_{i} \mathbf{E}_{i} J_{i}+\mathbf{E}_{0} W=0
$$


This is the famous $(0,2)$ supersymmetry constraint.

A superpotential specified by $\mathbf{E}_{i}, J_{i}$ leads to the same IR physics as one specified by $\mathbf{E}_{i}^{\prime}$, $J_{i}^{\prime}$ whenever the two are related by holomorphic field re-definitions. These re-definitions act on various $(0,2)$ multiplets and can be identified with the following sections:

$$
\begin{aligned}
\text { neutral chiral fields : } & H^{0}\left(\mathscr{O}_{V}^{\oplus r^{2}}\right), \\
\text { charged chiral fields : } & \oplus_{i} H^{0}\left(\mathscr{O}_{V}\left(\mathbf{q}_{i}\right)\right), \\
\text { charged Fermi fields : } & \oplus_{i, j} H^{0}\left(\mathscr{O}_{V}\left(\mathbf{q}_{i}-\mathbf{q}_{j}\right)\right) .
\end{aligned}
$$

These must of course be taken modulo gauge transformations and $\mathrm{U}(1)_{L}$ invariance. The data in E, $J$ and $W$ encodes both bundle and polynomial Calabi-Yau deformations. As is familiar from the monomial-divisor mirror map, the latter are nicely described by toric geometry [40] of $V$ and the Newton polytope for $W$. In particular, a choice of $W$ fixes the polynomial complex structure moduli of the Calabi-Yau, as well as re-definitions of the charged matter fields modulo gauge invariance.

Supposing we have fixed the complex structure on $X$, we can ask about the remaining $(0,2)$ deformations and field re-definitions. The first order deformations $\delta \mathbf{E}^{i}$ and $\delta J_{i}$ fit into the first position (recall that the zeroth position is marked by the dotted line) of the complex

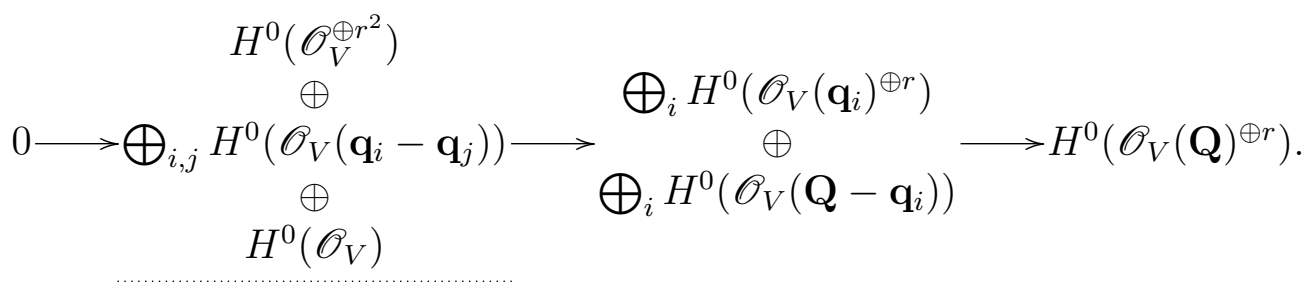

They must satisfy $\delta \mathbf{E}^{i} J_{i}+\mathbf{E}^{i} \delta J_{i}=0$.

This complex is just what we get for $H^{0}(\mathscr{H} o m(T, T))$, where $\mathscr{H} o m(T, T)$ is given in (55), but with two important differences. Firstly the sheaves relevant to the GLSM are defined over $V$, while those relevant to the geometric analysis are defined over $X$. This can lead to differences in the counting. For instance, in general $H^{0}\left(\mathscr{O}_{V}\left(\mathbf{q}_{i}-\mathbf{q}_{j}\right)\right) \neq H^{0}\left(\mathscr{O}_{X}\left(\mathbf{q}_{i}-\mathbf{q}_{j}\right)\right)$, and the latter can have additional holomorphic sections. When this holds, the GLSM superpotential modulo holomorphic field re-definitions over-parametrizes the bundle deformations, since there are automorphisms of the NLSM that cannot be lifted to holomorphic re-definitions of the UV theory. We will see an example of this phenomenon in the septic. Secondly, and more obviously, we are missing the first two terms of (55). These will vanish on $V$ but there are examples (not in this paper) where the restriction to $X$ can give nonzero entries.

We should also note another subtlety in the comparison: there is a difference between counting first order solutions to the supersymmetry constraint (65) and demanding that it is satisfied to all orders. The latter leads to GLSM deformations, while the former corresponds to massless states accessible via the GLSM. 


\subsection{Mapping Geometry to the Landau-Ginzburg Theory}

It is interesting to compare the geometric computation of the number of singlets with the Landau-Ginzburg description. At a crude level we know that the numbers agree, but can we make a more precise map? We can make some way via a series of exact sequences as follows. We restrict attention to the case $r=1$, i.e., there is only one $\mathrm{U}(1)$ charge.

Define $\mathscr{A}$ by

$$
0 \longrightarrow \mathscr{A} \longrightarrow \bigoplus_{i} \mathscr{O}_{X}\left(\mathbf{q}_{i}\right) \stackrel{\partial_{i} W}{\longrightarrow} \mathscr{O}_{X}(\mathbf{Q}) \longrightarrow 0 .
$$

(45) gives an exact sequence

$$
0 \longrightarrow \mathscr{O}_{X} \longrightarrow \mathscr{A} \longrightarrow T \longrightarrow 0
$$

From these sequences, the following is exact:

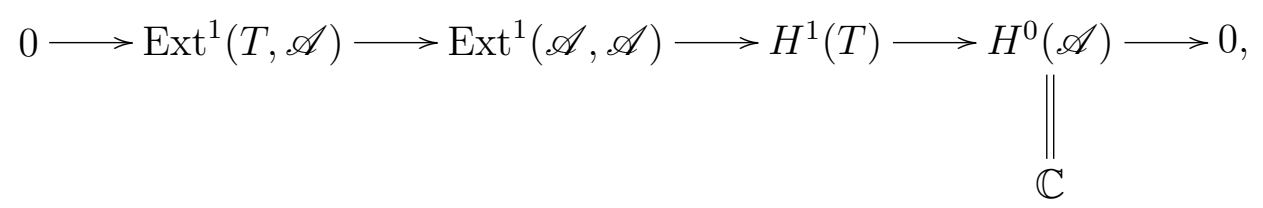

as is

$$
0 \longrightarrow H^{2}(T) \longrightarrow \operatorname{Ext}^{1}(T, \mathscr{A}) \longrightarrow \operatorname{Ext}^{1}(T, T) \longrightarrow 0 .
$$

These exact sequences show how the three sources of singlets, $H^{1}(T), H^{2}(T)$ and $\operatorname{Ext}^{1}(T, T)$ combine into $\operatorname{Ext}^{1}(\mathscr{A}, \mathscr{A})$ (minus one).

Now let $L_{1}$ and $L_{3}$ be the "universal" contributions from the Landau-Ginzburg theory at $k=1$ and $k=3$ respectively from table 3 . That is

$$
0 \longrightarrow H^{0}(\mathscr{O}) \longrightarrow \stackrel{\bigoplus_{i} H^{0}\left(\mathscr{O}\left(\mathbf{q}_{i}\right)\right)}{\bigoplus_{i, j} H^{0}\left(\mathscr{O}\left(\mathbf{q}_{i}-\mathbf{q}_{j}\right)\right)} \longrightarrow \bigoplus_{i} H^{0}\left(\mathscr{O}\left(\mathbf{Q}-\mathbf{q}_{i}\right)\right) \longrightarrow L_{1} \longrightarrow 0
$$

is exact, and $L_{3}=\bigoplus_{i} H^{0}\left(\mathscr{O}\left(\mathbf{q}_{i}\right)\right)$. These statements are written for the toric variety $V$ but one can show, in the case $r=1$, that they are also valid on restriction to $X$.

The relationship between $L_{1}, L_{3}$ and $\operatorname{Ext}^{1}(\mathscr{A}, \mathscr{A})$ is expressed by the fact that the following two sequences are exact (for generic $W$ ):

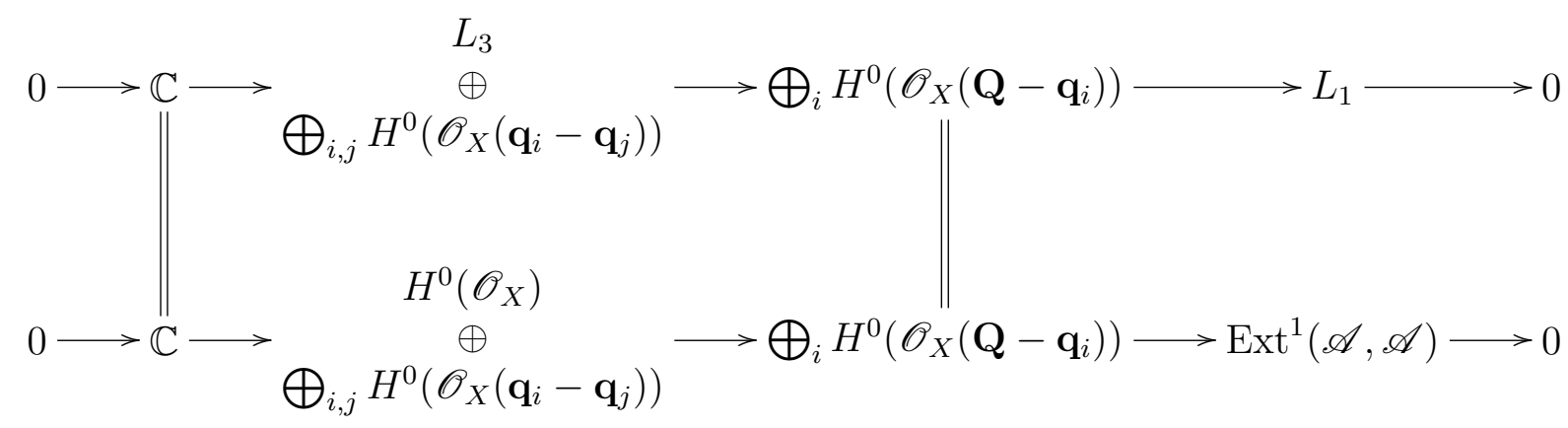


It is easy to see from this that dimensions are correct, i.e., the singlet count between the Landau-Ginzburg picture and the large radius picture agree:

$$
\operatorname{dim} L_{1}+\operatorname{dim} L_{3}=\operatorname{dim} H^{1}(T)+\operatorname{dim} H^{2}(T)+\operatorname{dim} H^{1}(\operatorname{End}(T)),
$$

but we also see that the precise mapping of singlets between these pictures is quite subtle.

\subsection{Orbifolds and a $(0,2)$ McKay Correspondence}

Phases in which $X$ acquires orbifold singularities are an interesting intermediate situation between the large-radius geometric phase and the Landau-Ginzburg phase. Near an orbifold limit point we can distinguish untwisted "bulk" states from twisted states localized near the singular locus. Deep in the orbifold phase, when all sizes in $X$ other than the cycle whose shrinking is responsible for the singularity are taken large, the geometry near the singular locus tends to a limit in which the space transverse to the singular locus is simply $\mathbb{C}^{d-D} / \Gamma$ for a quotient group $\Gamma$ and a singular locus of dimension $D$. In the orbifold limit, the theory acquires a discrete quantum symmetry, and states can be classified by their transformation properties under it. Invariant states, also termed untwisted states, correspond to strings occupying the "bulk" of $X$. Charged, or "twisted" states represent strings localized near the singular locus. The spectrum of massless twisted states can be determined from the local structure of $X$ near the singular locus.

When $X$ is a hypersurface in a toric variety, the GLSM provides a simple description of the untwisted sector. Deep in the orbifold phase, some of the chiral fields acquire large expectation values, breaking the gauge group down to a subgroup $\mathrm{U}(1)^{r^{\prime}} \times \Gamma, r^{\prime}<r$. Fluctuations of these fields acquire large masses through the Higgs mechanism ${ }^{10}$, and integrating them out we find an effective theory of the remaining chiral fields interacting with $r^{\prime}$ gauge multiplets and via an effective superpotential $\hat{W}$. Applying the GLSM picture of section 4.1 to this reduced model produces singlets in the untwisted sector of the model.

To describe the twisted sector we use the fact that twisted states are localized near the singular locus. This allows us to use the local geometry to find a free-field description near a point on the singular locus following [13]. For the reader's convenience we recall the analysis, recast in the notation we use here, restricting attention for simplicity to the case $\Gamma=\mathbb{Z}_{p}$. Near a point on the singular locus we pick local coordinates $x_{i}, i=1 \ldots 3$, on which the $\Gamma$ action is generated by $x_{i} \mapsto e^{2 \pi i n_{i} / p} x_{i}$ with $\sum n_{i}=0(\bmod p)$, and consider a free theory with chiral supermultiplets $\Phi_{i}$ and this (non- $R$ ) action. The twisted sectors of this orbifold of a free field theory, our approximation to the twisted sectors of the orbifold phase of $X$, can be described using the techniques of Section 2 simply setting $W=0$ and $\alpha_{i}=0$. We find twisted sectors labeled by $(k, s)$ where $k=1, \ldots p-1$, and $s=0,1$ distinguishes $\mathrm{R}$ $(s=0)$ sectors from NS $(s=1)$ boundary conditions on the left-moving fermions. In the

\footnotetext{
${ }^{10}$ As usual, we gloss over the subtleties of this in two dimensions.
} 
sector $(k, s)$ the boundary conditions on the fields are given by

$$
\begin{array}{rlrl}
\nu_{i} & =\frac{k n_{i}}{p} \quad(\bmod 1) & 0<\nu_{i} \leq 1 \\
\widetilde{\nu}_{i}=\frac{k n_{i}}{p}-\frac{s}{2}(\bmod 1) & -1<\widetilde{\nu}_{i} \leq 0
\end{array}
$$

while $h_{i}=0$ and $\widetilde{h}_{i}=\frac{1}{2}$. With $W=0$ computing $\bar{Q}$ cohomology is trivial, but we need to perform the projection onto $\Gamma$-invariant states, as well as the GSO projection.

The quotient preserves the four worldsheet supercharges given to within overall factors by

$$
G^{-}=\sum_{i} \gamma_{i} \partial \bar{x}_{i}, \quad G^{+}=\sum_{i} \bar{\gamma}_{i} \partial x_{i}, \quad \bar{G}^{-}=\sum_{i} \psi_{i} \bar{\partial} \bar{x}_{i}, \quad \bar{G}^{+}=\sum_{i} \bar{\psi}_{i} \bar{\partial} x_{i}
$$

The superscripts label the charges under the fermion number currents given by

$$
\begin{array}{ll}
J=\sum_{i} J_{i}, & J_{i}=\bar{\gamma}_{i} \gamma_{i} \\
\bar{J}=\sum_{i} \bar{J}_{i}, & \bar{J}_{i}=\bar{\psi}_{i} \psi_{i}
\end{array}
$$

The case $D=0$ - an isolated singular point - is simplest. We study an example in section 5.2. The free field theory exhibits (in general) an unbroken $\mathrm{E}_{6}$ gauge symmetry, and the methods we presented lead to predictions for the massless spectrum in twisted sectors. We find $\mathbf{2 7}$ and $\overline{\mathbf{2 7}}$ multiplets and their conjugates along with the $\mathrm{E}_{6}$ singlets related to them by the left-moving supersymmetry which are $(2,2)$ moduli. These are the subject of the McKay correspondence. In our conventions, chiral 27s correspond to Kähler deformations, while chiral $\overline{\mathbf{2 7}}$ s correspond to deformations of complex structure. There will also be $\mathrm{E}_{6}$ singlets in the twisted sectors, for which one can attempt to find an analogous correspondence. Note that the conjugate states will arise in the conjugate sector, so that in general some of these singlet states might be lifted in pairs by deformations resolving the singularity.

In the case $D=1$ - a curve $C$ of singularities - we proceed in stages. Near a point on $C$ we can find local coordinates as above, such that $n_{1}=0$. There are bosonic $x_{1}$ zero modes in the twisted sectors, so our states will locally be described by functions of $x_{1}$. A novel feature of this construction will be that excluding $x_{1}$ means the zero mode of $\bar{\psi}_{1}$ is no longer $\bar{Q}$-exact, and we will need to include it in our computations (recall that we compute in the right-moving $\mathrm{R}$ sector where $\psi_{1}$ satisfies the same untwisted boundary conditions as $x_{1}$ ). The free-field quotient preserves an unbroken $\mathrm{E}_{7}$ gauge symmetry given by the embedding $\mathrm{E}_{7} \times \mathrm{SU}(2) \subset \mathrm{E}_{8}$

We can then consider this quotient structure fibered over the (assumed to be) large curve $C$. The low-energy physics will be described by a nonlinear sigma model on $C$ with the fields determined from the orbifold construction as above. These will couple to the spin

\footnotetext{
${ }^{11}$ Pedants will note the missing quotient by $\mathbb{Z}_{2}$ here.
} 


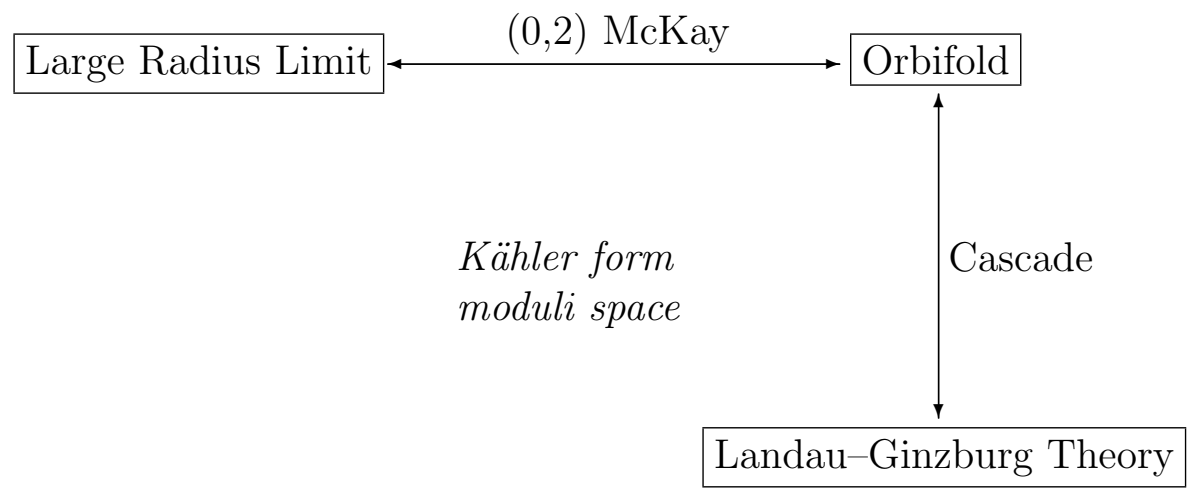

Figure 1: Mapping between Calabi-Yau, Landau-Ginzburg and orbifold phases.

connection on $C$ so that the functions of $x_{1}$ become sections of appropriate bundles. To find the spin we note that the curvature of $C$ breaks $\mathrm{E}_{7} \rightarrow \mathrm{E}_{6}$. Since this fits into the maximal embedding $\mathrm{E}_{6} \times \mathrm{SU}(3) \subset \mathrm{E}_{8}$ we see that $\mathrm{U}(1)_{C}$ must be an $\mathrm{SU}(3)$ subgroup. The fact that it must also commute with an $\mathrm{SU}(2)$ subgroup acting in the transverse directions (before taking the quotient) determines

$$
J_{C}=-J_{1}-\bar{J}_{1}+\frac{1}{2}\left(J_{2}+J_{3}+\bar{J}_{2}+\bar{J}_{3}\right)
$$

Simply compactifying the quotient theory on the curve $C$ will not lead to a supersymmetric spectrum. In fact, the local structure is not described as $C \times\left(\mathbb{C}^{2} / \Gamma\right)$ but as a fibration over $C$ such that the resulting space is Calabi-Yau. In terms of our fields, the transverse coordinates $x_{i}$ for $i \neq 1$ transform under $J_{C}$ in such a way that two of the supercharges are invariant. Moreover, the zero mode of $\bar{\psi}_{1}$, lying in the cokernel of $\bar{Q}$ in the nonlinear sigma model, becomes a section of $\Omega$, the cotangent bundle of $C$. When acting on a vertex operator corresponding to a space-time fermion in a massless chiral multiplet (with $\bar{q}=-\frac{1}{2}$ ) $\bar{\psi}_{1}$ will create the vertex operator for a massless antichiral multiplet $\left(\bar{q}=\frac{1}{2}\right)$. Thus fields in the nonlinear theory with charge $\bar{q}=-\frac{1}{2}$ and spin $q_{C}$ will produce massless chiral multiplets corresponding to $H^{0}\left(C, \Omega^{\otimes q_{C}}\right)$. Fields with charge $\bar{q}=\frac{1}{2}$ and spin $q_{C}$ will produce massless antichiral multiplets corresponding to $H^{1}\left(C, \Omega^{\otimes\left(q_{C}+1\right)}\right)$. CPT invariance, the requirement that each chiral multiplet of charge $q_{C}$ be accompanied by an antichiral multiplet of charge $-q_{C}$ is then tantamount to Serre duality on $C$.

This gives a matching between counting singlets in the orbifold language and the large radius language which works almost perfectly. Some interesting subtleties concerning dependence on complex structure will be seen in section 5.3.2.

The correspondence between the Landau-Ginzburg picture and the large radius picture proceeds typically as shown in figure 1. The orbifold corresponds to a weighted projective space where we essentially just consider a single $\mathbb{C}^{*}$-action (i.e., $r^{\prime}=1$ ), and so the results of section 4.2 apply. That is, we may relate the Landau-Ginzburg picture to the orbifold picture using the $L_{1}$ and $L_{3}$ contributions to the cascade. Then the orbifold may be related to the large radius limit by using the above $(0,2)$-McKay correspondence. 


\section{$5 \quad$ Examples}

\subsection{The sextic in $\mathbb{P}_{\{2,1,1,1,1\}}^{4}$}

\subsubsection{Geometry}

The weighted projective space $\mathbb{P}_{\{2,1,1,1,1\}}^{4}$ with homogeneous coordinates $\left[x_{0}, \ldots, x_{4}\right]$ has a terminal singularity at $x_{1}=x_{2}=x_{3}=x_{4}=0$. If $X$ is a generic hypersurface of degree 6 then it will not intersect this singularity. The Hodge numbers of $X$ are $h^{1,1}(X)=1$ and $h^{2,1}(X)=103$. The analysis of $H^{1}(X, \operatorname{End}(T))$ looks quite similar to that of the quintic. The spectral sequence is

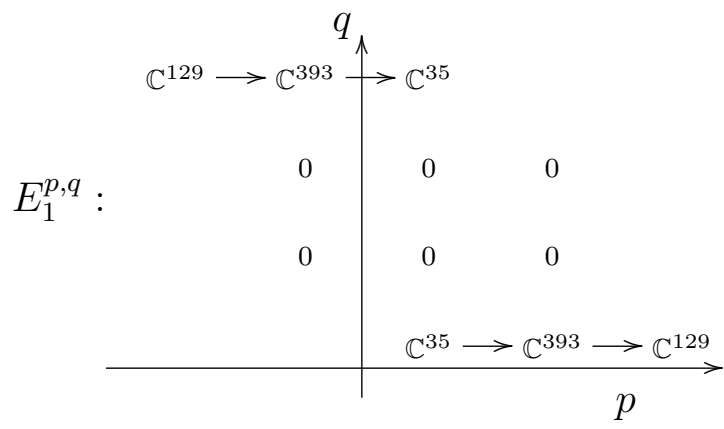

This gives $\operatorname{dim} H^{1}(X, \operatorname{End}(T))=230$. As in the case of the quintic, each of these corresponds to a $(0,2)$ GLSM deformation. Adding in singlets from $h^{1,1}, h^{2,1}$ and $\mathrm{U}(1)$ partners we would predict a value for the Gepner model of $230+1+103+4=338$. The actual value as given in the table of [7] is 344 . We are short by 6 .

\subsubsection{The Landau-Ginzburg locus}

The superpotential is a degree 6 polynomial with weights $\alpha_{0}=\frac{1}{3}$ and all other $\alpha_{i}=\frac{1}{6}$ (we take $i=1, \ldots, 4)$. Working sector by sector, we find the following zero energy states with $q=0$.

$$
\begin{array}{cc}
\bar{q}=-\frac{3}{2} & \bar{q}=-\frac{1}{2} \\
\gamma_{0} \bar{\gamma}_{0}|1\rangle_{1} \oplus \gamma_{i} \bar{\gamma}_{j}|1\rangle_{16} & \\
\oplus F_{[1] \gamma_{i} \bar{\gamma}_{0}|1\rangle_{16}} & F_{[4]} \gamma_{0}|1\rangle_{46} \\
\oplus & \oplus_{[5]}^{i} \gamma_{i}|1\rangle_{320} \\
G_{[1]}^{i} \rho_{i}|1\rangle_{16} \oplus H_{[2]} \rho_{0}|1\rangle_{11} & \\
& x_{i} \bar{\gamma}_{j}|3\rangle_{16} \oplus F_{[2]} \bar{\gamma}_{0}|3\rangle_{11} \\
& \\
& \rho_{0} \bar{\gamma}_{i} \bar{\gamma}_{j}|5\rangle_{6}
\end{array}
$$


In the $k=1$ sector $\bar{Q}: U_{-3 / 2} \rightarrow U_{-1 / 2}$ has a one-dimensional kernel for generic $W$; the dimension increases to 5 at the Fermat point. This is just what we have already observed in the quintic. So, we find $307 k=1$ chiral singlets for generic $W$ and 4 more at the Fermat point.

The $k=3$ states arise from the "cascade" picture described above, while the six $k=5$ states account for the discrepancy with geometry. As we will see, mirror symmetry shows that these states are lifted once we turn on the Kähler modulus to move away from the Landau-Ginzburg point.

\subsubsection{Mirror Symmetry and Kähler Deformations}

The Gepner model in this moduli space is given by a (quotient of) the product $A_{2} \oplus A_{5}^{\oplus 4}$ of minimal models at level $k_{i}=4$ and $k_{0}=1$. In addition to the universal "cascade" states in the $k=1$ and $k=3$ sectors, there are six singlet states in the $k=5$ sector. The Gepner model enjoys a discrete $S_{6}$ symmetry which permutes these singlet states. The Kähler deformation does not break this symmetry, so all of these singlets will be lifted by the deformation or none will.

Explicitly, the six massless singlet states at $k=5$ are given by permutations of

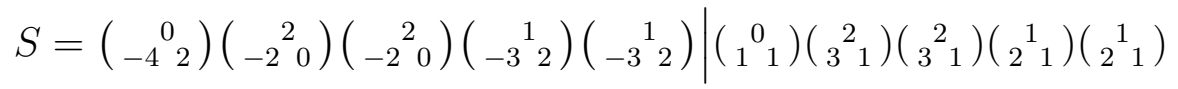

For clarity we have not cast this into standard form, exhibiting it in a way that makes the twist manifest.

The mirror model is given by a $\mathbb{Z}_{6}^{2} \times \mathbb{Z}_{3}$ quotient represented by the vectors

$$
m^{(1)}=(0,1,0,0,-1) \quad m^{(2)}=(0,0,1,0,-1) \quad m^{(3)}=(1,0,0,0,-2) .
$$

The state mirror to $S$ (obtained by reversing the signs of $q$ and $s$ ) satisfies

$$
\bar{q}=q+m^{(1)}+m^{(2)}-m^{(3)}-m^{(0)},
$$

where $m^{(0)}=(1,1,1,1,1)$, and so will appear in the sector $(k ; t)=(11 ; 1,1,2)$. Note that while the mirror model obviously shares the permutation symmetry of the original, this is not evident in its presentation as a quotient. Thus the states related to $S$ by permutations will arise in other twisted sectors.

In the Landau-Ginzburg model the discrete group acts via

$$
w^{1}=\left(0, \frac{1}{6}, 0,0,-\frac{1}{6}\right) \quad w^{2}=\left(0,0, \frac{1}{6}, 0,-\frac{1}{6}\right) \quad w^{3}=\left(\frac{1}{3}, 0,0,0,-\frac{1}{3}\right) .
$$

Constructing the Landau-Ginzburg orbifold we find in this sector the following states at $q=0$ :

$$
\begin{gathered}
\bar{q}=-\frac{1}{2} \\
\bar{q}=\frac{1}{2} \\
\bar{\gamma}_{0} x_{1}^{2} x_{2}^{2} \rho_{3}^{2} \rho_{4}^{2}|v\rangle \longrightarrow x_{1}^{3} x_{2}^{3} \rho_{3} \rho_{4}|v\rangle
\end{gathered}
$$


where $|v\rangle$ is the twisted vacuum. To find the action of $\bar{Q}$ note that since $\widetilde{\nu}_{0}=0$, the only term that can possibly contribute is $\bar{\gamma}_{0}^{\dagger}\left(\partial_{0} W\right)_{0}$. At the Gepner point $\partial_{0} W=2 x_{0}^{2}$, and expanding this we find that the $\bar{q}=-\frac{1}{2}$ state contributes to the cohomology at the Gepner point.

The mirror Landau-Ginzburg model has a unique superpotential deformation (related by mirror symmetry to the Kähler deformation of the original model). This corresponds to adding to the Fermat superpotential the unique monomial invariant under the quotient group:

$$
\delta W=\psi x_{0} x_{1} x_{2} x_{3} x_{4}
$$

This modifies $\bar{Q}$ as found above, introducing a term $\bar{\gamma}_{0}^{\dagger} \psi x_{1} x_{2} x_{3} x_{4}$, which upon expansion gives

$$
\delta \bar{Q}=\cdots+\psi \bar{\gamma}_{0}^{\dagger} x_{1} x_{2} \rho_{3}^{\dagger} \rho_{4}^{\dagger}
$$

rendering the kernel trivial. The state $S$, and thus all six $k=5$ singlets states found above, are lifted for $\psi \neq 0$ by a Kähler dependent mass term.

\subsection{The septic in $\mathbb{P}_{\{3,1,1,1,1\}}^{4}$}

\subsubsection{Geometry}

Let $X_{\text {orb }}$ be a septic hypersurface in $\mathbb{P}_{\{3,1,1,1,1\}}^{4}$. This weighted projective space has a codimension 4 quotient singularity but the degree of the hypersurface forces $X_{\text {orb }}$ to pass through this point. $X_{\text {orb }}$ thus has an isolated singularity of the form $\mathbb{C}^{3} / \mathbb{Z}_{3}$.

The polytope in the $N$ lattice associated with $\mathbb{P}_{\{3,1,1,1,1\}}^{4}$ is not reflexive but we may analyze the septic hypersurface in the general setup of the gauged linear $\sigma$-model in the following way. Let $P^{\circ}$ be the Newton polytope in $M$ for septics in $\mathbb{P}_{\{3,1,1,1,1\}}^{4}$. The 8 vertices are given by the equation

$$
x_{0}^{2} x_{1}+x_{0}^{2} x_{2}+x_{0}^{2} x_{3}+x_{0}^{2} x_{3}+x_{1}^{7}+x_{2}^{7}+x_{3}^{7}+x_{3}^{7} .
$$

Then define $P \subset N$ as the polar polytope. $P$ has 6 vertices, which may be taken to be $(1,0,0,0),(0,1,0,0),(0,0,1,0),(0,0,0,1),(-3,-1,-1,-1)$ and $(-1,0,0,0)$.

One then constructs a toric variety $V$ associated to $P$ with following data. The homogeneous coordinate ring is $R=\left[x_{0}, \ldots, x_{5}\right]$, the charges are given by

$$
\Phi=\left(\begin{array}{cccccc}
0 & 1 & 1 & 1 & 1 & -3 \\
1 & 0 & 0 & 0 & 0 & 1
\end{array}\right)
$$

and let $B$ be $\left(x_{0}, x_{5}\right)\left(x_{1}, x_{2}, x_{3}, x_{4}\right) . V$ is then a $\mathbb{P}^{1}$-bundle over $\mathbb{P}^{3} . X$ is a smooth Calabi-Yau hypersurface in this. The vertices of the Newton polytope correspond to the equation

$$
x_{0}^{2} x_{1}+x_{0}^{2} x_{2}+x_{0}^{2} x_{3}+x_{0}^{2} x_{3}+x_{1}^{7} x_{5}^{2}+x_{2}^{7} x_{5}^{2}+x_{3}^{7} x_{5}^{2}+x_{3}^{7} x_{5}^{2} .
$$

The divisor $x_{5}=0$ corresponds to a $\mathbb{P}^{2} \subset X$ with normal bundle $\mathscr{O}(-3)$ which arises as the exceptional divisor of the resolution of the $\mathbb{C}^{3} / \mathbb{Z}_{3}$ quotient singularity in $X_{\text {orb }}$. One easily computes $h^{1,1}(X)=2$ and $h^{2,1}(X)=122$. 
$X$ has an interesting relation with the Calabi-Yau threefold $X^{\prime}$, the resolution of the degree 14 hypersurface in $\mathbb{P}_{\{7,2,2,2,1\}}^{4}$ as first observed in [41]. One may follow extremal transitions between hypersurfaces in toric varieties [42] by shrinking the Newton polytope and thus growing its polar. That is, one drops terms in the defining equation corresponding to vertices of the convex hull of $P^{\circ}$. Typically this makes $X$ singular. The fact that $P$ grows corresponds to a resolution of singularities which allows $X$ to pass through an extremal transition. We may try to do the same thing with our septic by shrinking the convex hull of (90) to that of

$$
x_{0}^{2} x_{1}+x_{1}^{7} x_{5}^{2}+x_{2}^{7} x_{5}^{2}+x_{3}^{7} x_{5}^{2}+x_{3}^{7} x_{5}^{2} .
$$

The polar of this Newton polytope corresponds to $\mathbb{P}_{\{7,2,2,2,1\}}^{4}$ and thus $X$ appears to have undergone an extremal transition to $X^{\prime}$. That said, the defining equation (91) is actually smooth. The supposed transition is not a transition at all and $X^{\prime}$ is merely a smooth deformation of $X$.

Actually the septic $X$ should be considered more generic than the degree 14 hypersurface $X^{\prime}$ in the following sense. All 122 deformations of complex structure of $X$ are seen as polynomial deformations. For $X^{\prime}$, theorem 1 shows that 15 of the deformations are nonpolynomial. This is because $X^{\prime}$ has an exceptional divisor of the form $C \times \mathbb{P}^{1}$ where $C$ is a genus 15 curve. As observed in [43], a generic deformation of $X^{\prime}$ will break this divisor up into 28 rational curves. The latter geometry is seen in a generic $X$.

The analysis of $H^{1}(X, \operatorname{End}(T))$ proceeds as follows. The spectral sequence is

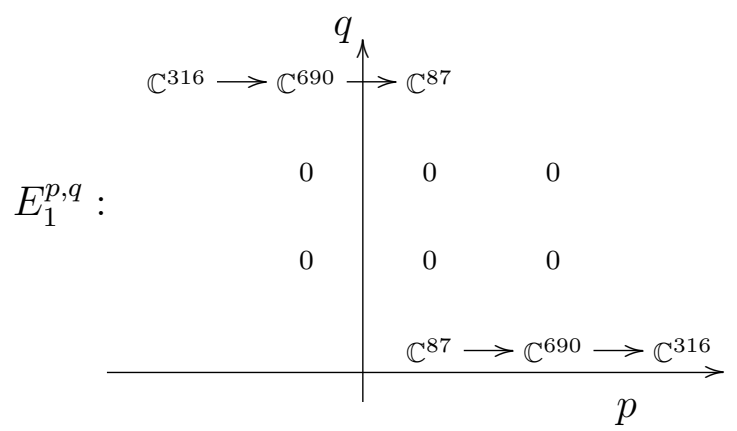

This has a new feature compared to the quintic and sextic of the previous sections. The $H^{0}$ of various line bundles computed in the bottom row of the spectral sequence must be computed on $X$ and not copied from $V$. For example $H^{0}\left(\mathscr{O}_{V}(4,-1)\right)$ is trivial while $H^{0}\left(\mathscr{O}_{X}(4,-1)\right)$ is dimension one.

Anyway, this gives $\operatorname{dim} H^{1}(X, \operatorname{End}(T))=288$. We can compare this to the Gepner model for $X^{\prime}$. Adding in singlets from $h^{1,1}, h^{2,1}$ and $\mathrm{U}(1)$ partners we would predict a value for the Gepner model of $288+2+122+3=415$, which is correct.

The counting of $(0,2)$ GLSM deformations following [9] naïvely yields 292 parameters associated to $H^{1}(X, \operatorname{End}(T))$. This is due to the four extra automorphisms counted by $H^{0}\left(\mathscr{O}_{X}(4,-1)\right)$ that cannot be lifted to the GLSM. Modulo this subtlety, we expect that all of the $(0,2)$ singlets identified by the geometric computation can be integrated up to deformations of the $(0,2)$ superpotential. 


\subsubsection{The Orbifold}

As argued in section 4.3 , we may analyze $X_{\text {orb }}$ in terms of the toric picture of the weighted projective space. That is, we have a homogeneous coordinate ring $R=\mathbb{C}\left[x_{0}, \ldots, x_{4}\right]$ with the grading giving by the weights $(3,1,1,1,1)$ and the irrelevant ideal is simply $B=$ $\left(x_{0}, x_{1}, \ldots, x_{4}\right)$.

The spectral sequence of (49) which computes the cohomology of the tangent sheaf becomes

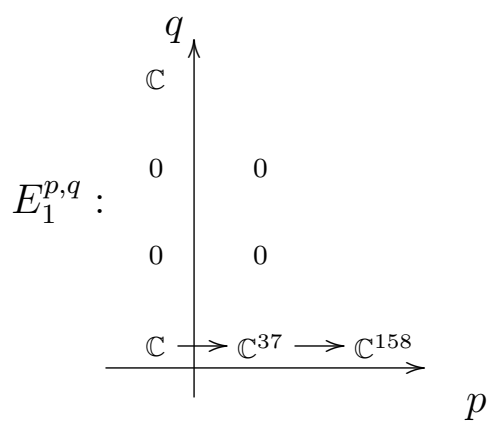

which predicts $h^{2,1}=122$ and $h^{1,1}=1$. Recall that these are the contributions from the untwisted sector of the orbifold. The value of $h^{2,1}$ is correct but we need to add one twisted state to $h^{1,1}$ to account for the $\mathbb{C}^{3} / \mathbb{Z}_{3}$ singularity. Then we agree with above.

The corresponding spectral sequence for $\operatorname{End}(T)$ gives

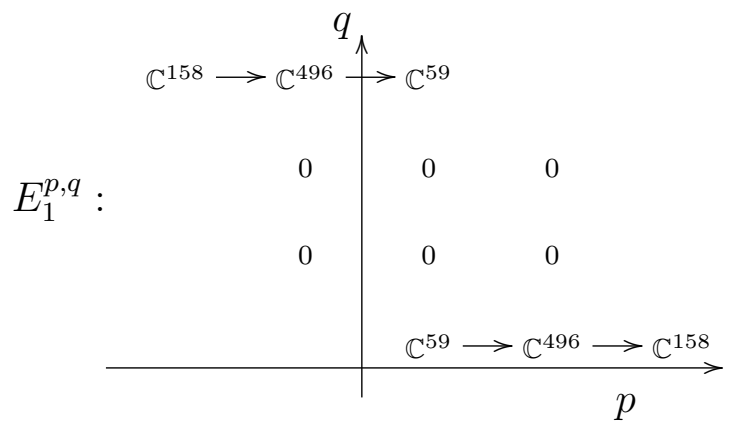

which yields $\operatorname{dim} H^{1}\left(X_{\text {orb }}, \operatorname{End}(T)\right)=280$. Comparing to above, we see that there must be 8 twisted stated to yield to the total of 288.

So we predict that there are 9 twisted singlets states -1 contributing to $h^{1,1}$ and 8 to $H^{1}(\operatorname{End}(T))$. Indeed the free-field calculation of $[13]$ reproduces this. We have here two twisted sectors $k=1,2$ and since they are related by conjugation we may restrict attention to $k=1$. These twisted vacua are invariant under $\mathbb{Z}_{3}$, i.e. $q_{g}$ of $(31)$ is zero.

In the $(k, s)=(1,0)$ (twisted R) sector we find $\nu_{i}=\frac{1}{3}, \widetilde{\nu}_{i}=-\frac{2}{3}$. As usual $E_{(1,0)}=0$, and the vacuum charges are $\left(q_{(1,0)}, \bar{q}_{(1,0)}\right)=\left(\frac{1}{2},-\frac{1}{2}\right)$. There are no zero modes in the internal theory, so the unique R ground state leads (after GSO projection) to chiral fermions in the $1 \mathbf{6}_{1 / 2}$ of $\mathrm{SO}(10)$. 
In the $(1,1)$ (twisted NS) sector we have $\nu_{i}=\frac{1}{3}, \widetilde{\nu}_{i}=-\frac{1}{6}$. Here $E_{(1,1)}=-\frac{1}{2}$ and the vacuum charges are $\left(q_{(1,1)}, \bar{q}_{(1,1)}\right)=\left(-1,-\frac{1}{2}\right)$. There are thus massless states in the $\mathbf{1 0}_{-1}$. Corresponding to these is the associated singlet (Kähler modulus) given by $G^{+}|1,1\rangle$. Internal excitations yield the nine massless singlet states $x_{i} \bar{\gamma}_{j}|1,1\rangle$ with charge $\left(0,-\frac{1}{2}\right)$ of which one linear combination is the Kähler modulus mentioned above, as well as the state $\bar{\gamma}_{1} \bar{\gamma}_{2} \bar{\gamma}_{3}|1,1\rangle$ with charges $\left(2,-\frac{1}{2}\right)$.

We thus find a chiral 27 predicted by the McKay correspondence, as well as the 9 singlets predicted above.

\subsubsection{The Landau-Ginzburg analysis}

The Landau-Ginzburg phase of the corresponding GLSM is described by a degree 7 superpotential $W\left(x_{0}, \ldots, x_{4}\right)$, with $\alpha_{0}=\frac{3}{7}$ and $\alpha_{i}=\frac{1}{7}$ for $i=1, \ldots, 4$. The zero energy states with $q=0$ are given by

$$
\begin{aligned}
& \bar{q}=-\frac{3}{2} \quad \bar{q}=-\frac{1}{2} \quad \bar{q}=\frac{1}{2} \\
& \gamma_{0} \bar{\gamma}_{0}|1\rangle_{1} \oplus \gamma_{i} \bar{\gamma}_{j}|1\rangle_{16} \\
& \begin{array}{c}
\oplus G_{[1]}^{j} \rho_{j}|1\rangle_{16} \\
\oplus
\end{array} \quad \begin{array}{c}
H_{[4]}^{0} \gamma_{0}|1\rangle_{39} \\
H_{[6]}^{i} \gamma_{i}|1\rangle_{420}
\end{array} \\
& H_{[2]}^{i 0} \gamma_{i} \bar{\gamma}_{0}|1\rangle_{40} \oplus G_{[3]}^{0} \rho_{0}|1\rangle_{21} \\
& \rho_{0} \bar{\gamma}_{i}|3\rangle_{4} \longrightarrow \bar{Q} \longrightarrow \begin{array}{l}
K_{[1]}^{j} \bar{\gamma}_{j} \gamma_{0}|3\rangle_{16} \\
\bigoplus \\
K_{[3]}^{0} \gamma_{0}|3\rangle_{21}
\end{array} \\
& \underset{x_{i} \bar{\gamma}_{j}|5\rangle_{16}}{x_{0} \bar{\gamma}_{0}|5\rangle_{1}} \longrightarrow x_{0}^{2} x_{i}|5\rangle_{4}
\end{aligned}
$$

Once again, for $k=1, \bar{Q}: U_{-3 / 2} \rightarrow U_{-1 / 2}$ has a one-dimensional kernel for $W$ generic and a five-dimensional kernel at the Gepner point, which corresponds to,

$$
W=\left(x_{0}^{2} x_{1}+x_{1}^{7}\right)+x_{2}^{7}+x_{3}^{7}+x_{4}^{7}
$$

i.e. the minimal model $D_{8} \oplus A_{6}^{\oplus 3}$. Adding up the states, we find $366 k=1$ chiral singlets for generic $W$.

In the $k=3$ sector, $\bar{Q}$ has a trivial kernel unless $W^{55}=W^{i 5}=0$, but that is a singular superpotential. So, this sector contributes $16+21-4=33$ singlets for any non-singular $W$. This model demonstrates the "cascade" described in 2.5. In the third sector we have in addition to the $\bar{q}=-\frac{1}{2}$ states listed in 28 the set of four states of charge $\bar{q}=-\frac{3}{2}$ 
and a nontrivial action of $\bar{Q}$ so that there are less massless singlets in this sector than the "universal" prediction. However, these states return in the $k=9$ sector (not shown) as four states of charge $\bar{q}=-\frac{1}{2}$ (related by conjugation to the four states at charge $\bar{q}=\frac{1}{2}$ at $k=5$ ).

Finally, we consider the $k=5$ sector. Writing $W$ as

$$
W=f_{[7]}\left(x_{i}\right)+g_{[4]}\left(x_{i}\right) x_{0}+s^{i} x_{i} x_{0}^{2},
$$

we see that the action of $\bar{Q}$ on an arbitrary state

$$
|\psi\rangle=\left(a x_{0} \bar{\gamma}_{0}+b^{i j} x_{i} \bar{\gamma}_{j}\right)|5\rangle \in U_{-1 / 2}
$$

is

$$
\bar{Q}|\psi\rangle=\left(2 a s^{i} x_{i}+s^{i} b^{j i} x_{j}\right) x_{0}^{2}|5\rangle .
$$

Thus, all of the $U_{1 / 2}$ states are $\bar{Q}$-exact for any non-singular $W$, and we find 13 chiral singlets in $k=5$. This total agrees with the orbifold and large radius phases.

\subsection{The octic in $\mathbb{P}_{\{2,2,2,1,1\}}^{4}$}

\subsubsection{Geometry}

The weighted projective space $\mathbb{P}_{\{2,2,2,1,1\}}^{4}$ with homogeneous coordinates $\left[x_{0}, \ldots, x_{4}\right]$ has a $\mathbb{Z}_{2}$ quotient singularity along $x_{0}=x_{1}=x_{2}=0$. This may be resolved to yield a toric variety $V_{0}$ with homogeneous coordinates $\left[x_{0}, \ldots, x_{5}\right]$, an irrelevant ideal $B=\left(x_{0}, x_{1}, x_{2}, x_{5}\right)\left(x_{3}, x_{4}\right)$ and grades given by the charge matrix

$$
\Phi=\left(\begin{array}{cccccc}
0 & 0 & 0 & 1 & 1 & -2 \\
1 & 1 & 1 & 0 & 0 & 1
\end{array}\right)
$$

$X_{0}$ is an octic hypersurface in $V_{0}$ with defining equation, in Fermat form,

$$
x_{0}^{4}+x_{1}^{4}+x_{2}^{4}+\left(x_{3}^{8}+x_{4}^{8}\right) x_{5}^{4} .
$$

Mirror symmetry was studied in detail for this example in [44].

The exceptional set in $X_{0}$ formed by the $\mathbb{Z}_{2}$-resolution is of the form $E=C \times \mathbb{P}^{1}$, where $C$ is a genus 3 curve. One has $h^{1,1}(X)=2$, where the two deformations of $B+i J$ can be considered to be the overall volume and a size of $E$. One may also show $h^{2,1}=86$. Of these 86 deformations of complex structure, 83 are obtained by deformations of the polynomial (101). The remaining 3 deformations of complex structure arise from $H^{1}\left(\mathscr{O}_{X}(-2,1)\right)=3$ in agreement with theorem 1. The 83 polynomial deformations of complex structure preserve $E=C \times \mathbb{P}^{1}$. We will see below that the remaining 3 deformations break $E$ apart into 4 disjoint $\mathbb{P}^{1}$ 's in accord with [43]. 
The $E_{1}$ stage of the spectral sequence to compute $H^{1}\left(X_{0}, \operatorname{End}(T)\right)$ is given by

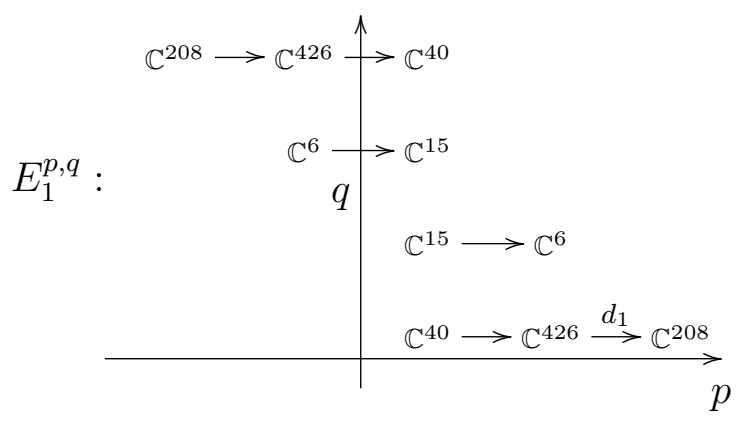

The map marked $d_{1}$ in 102 fails to be surjective in this case which makes for more interesting analysis compared to the above examples. Let $R_{4}$ denote the vector space of degree 4 polynomials in the variables $\left\{x_{0}, x_{1}, x_{2}\right\}$. Then one can show that

$$
\text { coker } d_{1}=\frac{R_{4}}{\left.\left(x_{0}, x_{1}, x_{2}\right)\left(\partial_{0} W, \partial_{1} W, \partial_{2} W\right)\right|_{x_{5}=0}} .
$$

This is a 6-dimensional space. In particular, for example, if $W$ is in Fermat form then coker $d_{1}$ is spanned by $\left\{x_{0}^{2} x_{1}^{2}, x_{0}^{2} x_{2}^{2}, x_{1}^{2} x_{2}^{2}, x_{0}^{2} x_{1} x_{2}, x_{0} x_{1}^{2} x_{2}, x_{0} x_{1} x_{2}^{2}\right\}$. The map on row one of the spectral sequence is shown to be surjective in the appendix.

If one were to replace the map $x_{i} \mathbf{q}_{i}$ in (45) with a generic map of the right multi-degree then the map $d_{1}$ in 102 becomes surjective. This means that a deformation to a more generic $(0,2)$-model kills any massless states that appear at the $(2,2)$-locus due to a failure of surjectivity of $d_{1}$. For this generic $(0,2)$-model the spectral sequence becomes degenerate at the $E_{2}$ stage:

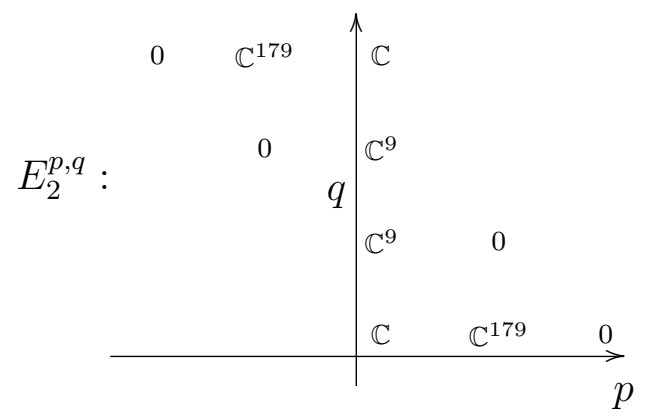

This yields a generic value of $\operatorname{dim} H^{1}\left(X_{0}, \operatorname{End}(T)\right)=188$.

However, on the $(2,2)$-locus, where $d_{1}$ fails to be surjective $H^{1}\left(X_{0}\right.$, End $(T)$ may jump to a higher value. A precise analysis of this is not too difficult, but the technical details may be a little distracting. So this computation is left to the appendix. The result is that for the Fermat polynomial one finds $\operatorname{dim} H^{1}\left(X_{0}, \operatorname{End}(T)\right)=200$, but that this value falls back to 
188 for a generic $\mathrm{W}$, even on the $(2,2)$-locus. This kind of jumping in $\operatorname{dim} H^{1}\left(X_{0}, \operatorname{End}(T)\right)$ as one varies the complex structure was seen in other examples in [5].

Three of the deformations of complex structure of $X_{0}$ are obstructed in the sense that they prevent $X_{0}$ being embedded in the toric variety we have considered so far. That said, it is still possible to understand these deformations in terms of a hypersurface in a toric variety as follows.

$V$ is the crepant resolution of the weighted projective space $\mathbb{P}_{\{2,2,2,1,1\}}^{4}$. It can be viewed as a $\mathbb{P}^{3}$-bundle over $\mathbb{P}^{1}$. To be precise, the toric data implies it is the space

$$
\mathbb{P}(\mathscr{O}(-2) \oplus \mathscr{O} \oplus \mathscr{O} \oplus \mathscr{O})
$$

over $\mathbb{P}^{1}$. The short exact sequence

$$
0 \longrightarrow \mathscr{O}(-2) \longrightarrow \mathscr{O}(-1)^{\oplus 2} \longrightarrow \mathscr{O} \longrightarrow 0,
$$

on $\mathbb{P}^{1}$ shows that $\mathscr{O}(-1)^{\oplus 2}$ may be viewed as a deformation of $\mathscr{O} \oplus \mathscr{O}(-2)$. That is, we may construct a family of line bundles over $\mathbb{P}^{1}$ where the central fibre is $\mathscr{O} \oplus \mathscr{O}(-2)$ and all other fibres are $\mathscr{O}(-1)^{\oplus 2}$. In the same way, there is a three-dimensional space of first-order deformations of complex structure that take $\mathbb{P}(\mathscr{O}(-2) \oplus \mathscr{O} \oplus \mathscr{O} \oplus \mathscr{O})$ into $\mathbb{P}\left(\mathscr{O}(-1)^{\oplus 2} \oplus \mathscr{O} \oplus \mathscr{O}\right)$.

So let $V_{1}$ be $\mathbb{P}\left(\mathscr{O}(-1)^{\oplus 2} \oplus \mathscr{O} \oplus \mathscr{O}\right)$ which we may define as a toric variety with homogeneous coordinate ring $R=\mathbb{C}\left[x_{0}, \ldots, x_{5}\right]$,

$$
\Phi=\left(\begin{array}{llllll}
1 & 1 & 1 & 1 & 0 & 0 \\
1 & 1 & 0 & 0 & 1 & 1
\end{array}\right)
$$

and $B=\left(x_{0}, x_{1}, x_{4}, x_{5}\right)\left(x_{2}, x_{3}\right)$. Let $X_{1}$ be a Calabi-Yau hypersurface. For a specific complex structure one might consider the defining equation

$$
W_{1}=x_{0}^{4}+x_{1}^{4}+x_{2}^{4} x_{4}^{4}+x_{2}^{4} x_{5}^{4}+x_{3}^{4} x_{4}^{4}+\lambda x_{3}^{4} x_{5}^{4},
$$

where $\lambda$ is a generic complex number (not equal to one or else the threefold is singular).

From what we have said, $X_{0}$ and $X_{1}$ are deformation equivalent. ${ }^{12}$ Putting $x_{4}=x_{5}=0$ in $X_{1}$ forces $x_{0}^{4}+x_{1}^{4}=0$ and thus yields 4 rational curves. These 4 rational curves in $X_{1}$ are what remains of the genus 3 curve times $\mathbb{P}^{1}$ in $X_{0}$ after switching on any of the three non-polynomial deformations of $X_{0}$.

Note that $X_{1}$ exhibits all 86 deformations of complex structure as polynomial deformations.

\footnotetext{
${ }^{12}$ One can also show that the A-model chiral rings of the $X_{0}$ and $X_{1}$ GLSMs are isomorphic: these models are describing the same moduli space of $(2,2)$ SCFTs.
} 
Let us compute $H^{1}\left(X_{1}\right.$, End $\left.(T)\right)$. The spectral sequence yields

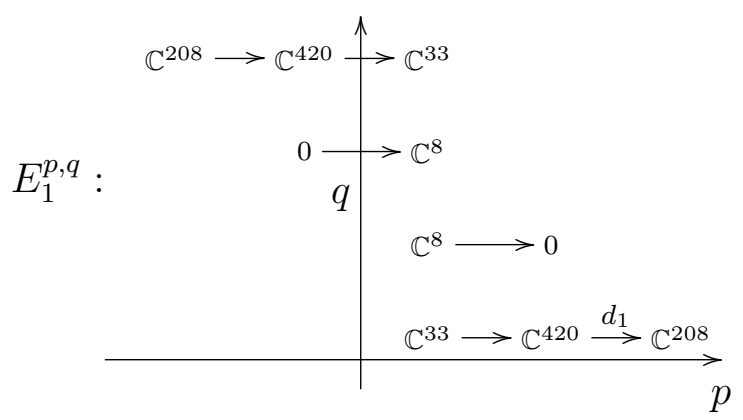

The map $d_{1}$ fails to be surjective, similarly to the $X_{0}$ case. The next stage of the spectral sequence is

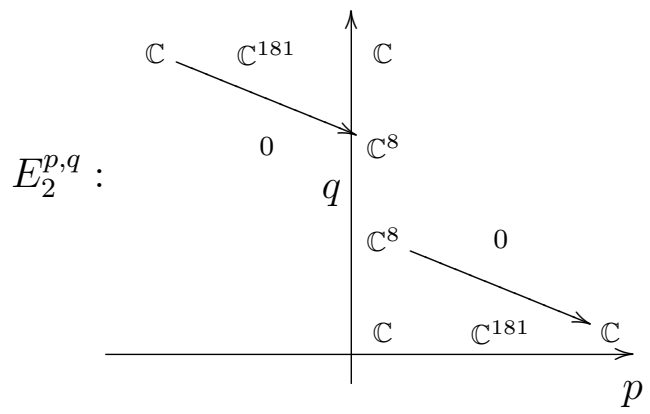

The $d_{2}$ maps in this spectral sequence may or may not be zero depending on the precise complex structure. The computation is very similar to that for $X_{0}$ in the appendix. The result is that $d_{2}$ is zero for the specific equation (108) but becomes nonzero for a generic defining equation. Thus

$$
\operatorname{dim} H^{1}\left(X_{1}, \operatorname{End}(T)\right)= \begin{cases}190 & \text { for the hypersurface } 108 \\ 188 & \text { generically }\end{cases}
$$

This fits in very nicely with the picture for $X_{0}$. Once again we have a generic value of 188 for $\operatorname{dim} H^{1}(X, \operatorname{End}(T))$ but this can increase for specific complex structures. $X_{1}$ is "more generic" than $X_{0}$ and cannot achieve as large a value for $\operatorname{dim} H^{1}(X, \operatorname{End}(T))$. The numbers of GLSM deformations for $X_{0}$ and $X_{1}$ are, respectively, 179 and 180.

\subsubsection{The Orbifold}

$X_{\text {orb }}$ is the singular octic in the unresolved weighted projective space $\mathbb{P}_{\{2,2,2,1,1\}}^{4}$. We may compute the untwisted sector easily enough in a way analogous to section 5.2 .2 . The result 
is that

$$
\begin{aligned}
h_{0}^{1,1} & =1 \\
h_{0}^{2,1} & =83 \\
\operatorname{dim} H^{1}(X, \operatorname{End}(T))_{0} & =182,
\end{aligned}
$$

where the subscript 0 denotes the untwisted sector.

$X_{\text {orb }}$ exhibits a genus 3 curve $C$ of singularities and $\Gamma=\mathbb{Z}_{2}$. We now have two twisted sectors with $k=1$, where $\nu_{1}=0$ and $\nu_{a}=\frac{1}{2}$ for $a=2,3$. Note that the $k=1$ sectors are self-conjugate, and (31) shows that the twisted vacua are $\mathbb{Z}_{2}$-invariant.

In the $(k, s)=(1,0)(\mathrm{R})$ sector we have $\widetilde{\nu}_{1}=0, \widetilde{\nu}_{a}=-\frac{1}{2} \cdot E_{(1,0)}=0$, and the vacuum charges are $\left(q_{(1,0)}, \bar{q}_{(1,0)}\right)=\left(-\frac{1}{2},-\frac{1}{2}\right)$. In this sector we have zero modes of $\gamma_{1}$ and $\psi_{1}$ so that the ground state is fourfold degenerate. Letting $|1,0\rangle$ denote the state annihilated by $\gamma_{1}$ and $\psi_{1}$, we have the four ground states with their associated charges and spins along $C,\left(q, \bar{q} ; q_{C}\right)$

$$
|1,0\rangle_{(-1 / 2,-1 / 2 ; 1)} \quad \bar{\gamma}_{1}|1,0\rangle_{(1 / 2,-1 / 2 ; 0)} \quad \bar{\psi}_{1}|1,0\rangle_{(-1 / 2,1 / 2 ; 0)} \quad \bar{\gamma}_{1} \bar{\psi}_{1}|1,0\rangle_{(1 / 2,1 / 2 ;-1)} .
$$

All of these are $\Gamma$-invariant and after GSO projection they lead to massless chiral supermultiplets with $\mathrm{SO}(10) \times \mathrm{U}(1)_{L} \times \mathrm{U}(1)_{C}$ transformation properties $\overline{\mathbf{1 6}}_{-1 / 2 ; 0} \oplus \mathbf{1 6}_{1 / 2 ; 1}$ along with their antichiral conjugates.

In the $(1,1)(\mathrm{NS})$ sector we have $\widetilde{\nu}_{1}=-\frac{1}{2}, \widetilde{\nu}_{a}=0$. Here $E_{(1,1)}=-\frac{1}{2}$, and the charges are $\left(q_{(1,1)}, \bar{q}_{(1,1)}\right)=\left(-1,-\frac{1}{2}\right)$. Here there are zero modes of $\gamma_{a}$ as well as $\bar{\psi}_{1}$ and hence the ground state is eightfold degenerate. With our usual notation we have

$$
\begin{array}{lll}
|1,1\rangle_{(-1,-1 / 2 ; 0)} & \bar{\gamma}_{2} \bar{\gamma}_{3}|1,1\rangle_{(1,-1 / 2 ; 1)} & \bar{\gamma}_{a}|1,1\rangle_{(0,-1 / 2 ; 1 / 2)} \\
\bar{\psi}_{1}|1,1\rangle_{(-1,1 / 2 ;-1)} & \bar{\psi}_{1} \bar{\gamma}_{2} \bar{\gamma}_{3}|1,1\rangle_{(1,1 / 2 ; 0)} & \bar{\psi}_{1} \bar{\gamma}_{a}|1,1\rangle_{(0,1 / 2 ;-1 / 2)}
\end{array}
$$

The first line will lead to chiral multiplets, the second to antichiral (after acting with leftmoving excitations). The two states listed last on each line are $\Gamma$-odd, the others $\Gamma$-even. The $\Gamma$-even ground states will lead to massless chiral multiplets transforming as $\mathbf{1 0}_{-1 ; 0} \oplus \mathbf{1 0}_{1 ; 1}$ and their antichiral conjugates. Acting with the supercharges we find the associated singlets

$$
\begin{aligned}
G^{+}|1,1\rangle & =\sum_{a} x_{a} \bar{\gamma}_{a}|1,1\rangle_{(0,-1 / 2 ; 0)} & \mathbf{1}_{0 ; 0} \\
G^{-} \bar{\gamma}_{2} \bar{\gamma}_{3}|1,1\rangle & =\left(\rho_{2} \bar{\gamma}_{3}-\rho_{3} \bar{\gamma}_{2}\right)|1,1\rangle_{(0,-1 / 2 ; 1)} & \mathbf{1}_{0 ; 1} .
\end{aligned}
$$

Internal excitations lead to additional GSO and $\Gamma$ invariant chiral states as listed below, with their $\mathrm{SO}(10) \times \mathrm{U}(1)_{L} \times \mathrm{U}(1)_{C}$ transformation properties, as well as the antichiral conjugates

$\begin{array}{lr}\gamma_{1}|1,1\rangle & \mathbf{1}_{-2 ; 1} \\ \bar{\gamma}_{1} \bar{\gamma}_{2} \bar{\gamma}_{3}|1,1\rangle & \mathbf{1}_{2 ; 0} \\ \gamma_{1} \bar{\gamma}_{2} \bar{\gamma}_{3}|1,1\rangle & \mathbf{1}_{0 ; 2} \\ \bar{\gamma}_{1}|1,1\rangle & \mathbf{1}_{0 ;-1} \\ x_{a} \bar{\gamma}_{b}|1,1\rangle_{4} & \mathbf{1}_{0 ; 0} \\ \rho_{a} \bar{\gamma}_{b}|1,1\rangle_{4} & \mathbf{1}_{0 ; 1}\end{array}$


Note that $x_{a}, \rho_{a}$ have spins $-\frac{1}{2}, \frac{1}{2}$, respectively.

Collecting all of this together into representations of $\mathrm{E}_{6} \times U(1)_{C}$ we find that the chiral multiplets fill out

$$
\mathbf{2} \mathbf{7}_{0} \oplus \overline{\mathbf{2 7}}_{1} \oplus \mathbf{1}_{-1} \oplus \mathbf{1}_{2} \oplus\left(\mathbf{1}_{1}\right)^{\oplus 4} \oplus\left(\mathbf{1}_{0}\right)^{\oplus 4}
$$

with the antichiral conjugates as expected. Note that one of the states in $\left(\mathbf{1}_{0}\right)^{\oplus 4}$ is related by $(115)$ to the $\mathbf{2} \mathbf{7}_{0}$ and one of the states in $\left(\mathbf{1}_{1}\right)^{\oplus 4}$ is related to the $\overline{\mathbf{2 7}}_{1}$.

When we compactify on $C$ we now find that massless chiral multiplets are given by holomorphic sections of $\Omega^{\otimes q_{C}}$. Riemann-Roch and vanishing theorems give

$$
h^{0}\left(\Omega^{\otimes q_{C}}\right)= \begin{cases}0 & q_{C}<0 \\ 1 & q_{C}=0 \\ g & q_{C}=1 \\ \left(2 q_{C}-1\right)(g-1) & q_{C}>1 .\end{cases}
$$

For $q_{C}=0$ we thus predict from the twisted sector one massless $\mathbf{2 7}$ and the single associated Kähler modulus, as well as 3 additional singlets regardless of the genus of $C$.

Putting $g=3$ for the case at hand we predict, from $q_{C}=1$ states, 3 massless $\overline{\mathbf{2 7}} \mathrm{s}$ with the associated 3 complex structure moduli, as well as an additional 9 singlets. We also find 6 massless singlets from the $q_{C}=2$ states.

Adding together the untwisted and twisted states we obtain

$$
\begin{aligned}
& h^{1,1}\left(X_{\text {orb }}\right)=1+1=2, \\
& h^{2,1}\left(X_{\text {orb }}\right)=83+3=86
\end{aligned}
$$

as expected. More interestingly, we have a grand total of 288 singlets. That is, we predict 200 singlets associated with $H^{1}(\operatorname{End}(T))$. This total agrees with the massless spectrum for large radius phase for the Fermat complex structure.

So we have agreement with the large radius (and Landau-Ginzburg phase - as we see shortly) only for particular values of the complex structure of $X_{0}$. In particular, our orbifold computation seems, at first sight, not to depend at all on the value of the complex structure of $X_{0}$. How can we resolve this discrepancy?

We do not know the full resolution of this question, but we can make the following observations. Our analysis in terms of free fields of the twisted sector effectively assumes that we were analyzing the normal bundle of $C$ in $X_{0}$ rather than $X_{0}$ itself. The deviations of the geometry away from the normal bundle as we move away from $C$ must introduce corrections that we have ignored so far.

Which states counted above do we believe are reliably massless? The fields in nontrivial $\mathrm{E}_{6}$ representations, along with the associated Kähler and complex structure moduli, are of course protected by the $(2,2)$ supersymmetry; in addition, the 6 singlet states coming from $q_{C}=2$ involve only excitations along $C$ and we do not expect them to be sensitive to the details of the structure of $X$ away from the curve. The 12 additional singlet states coming from $q_{C}=0,1$ involve excitations in the directions transverse to the curve. There is nothing 
to stop these states being lifted by the additional interactions introduced upon varying the superpotential. Thus we might reasonably expect there to be 12 fewer singlets for a generic complex structure. This agrees perfectly with the large radius picture.

\subsubsection{The Landau-Ginzburg locus}

Using the GLSM, it is easy to describe the Landau-Ginzburg point of the GLSM for $X_{0}$. Integrating out the $x_{5}$ and $p$ fields leads to a theory with fields $x_{0}, \ldots, x_{4}$, with $\alpha_{0,1,2}=\frac{1}{4}$, and $\alpha_{3,4}=\frac{1}{8}$. Assigning weights $[2,2,2,1,1]$ to the fields, the Landau-Ginzburg superpotential is a degree 8 polynomial in 5 variables which we denote

$$
\left.\widetilde{W}\left(x_{0} \ldots x_{4}\right) \equiv W\right|_{x_{5}=1} .
$$

We will distinguish the fields with different $\alpha_{i}$ by taking indices $I, J=0,1,2$, and $a, b=3,4$.

With these numbers in hand, we classify the zero energy states with $q=0$ as shown in table 4 .

For generic $\widetilde{W}$ we will have 244 singlets from $k=1$, while for Fermat we find 248. As in the example of the quintic, the physical reason for the appearance of these extra singlets is just the Higgs mechanism.

The $k=3$ and $k=5$ zero energy states clearly satisfy $\bar{Q}=0$, so that all of these correspond to massless singlets.

The $k=9$ sector states are the CPT conjugates of the states at $k=7$ and the $\bar{Q}$ complex is the transpose of the complex for $k=7$. We can thus equivalently study either sector, and since the structure at $k=9$ more transparently reflects the description in the previous subsection we choose this option. The antichiral multiplets from this sector (conjugate to the chiral multiplets at $k=7$ ) are determined by the cokernel of $\bar{Q}$. Using $\nu_{I}=\frac{1}{8}$ and $\nu_{a}=\frac{9}{16}$ and hence $\widetilde{\nu}_{I}=-\frac{3}{8}, \widetilde{\nu}_{a}=-\frac{15}{16}$, the map in this sector is determined by the following terms

$$
\begin{aligned}
& \bar{Q}_{1}=\bar{\gamma}_{I}^{\dagger}\left(\partial_{I} \widetilde{W}\right)_{\widetilde{\nu}_{I}}=\left.\bar{\gamma}_{I}^{\dagger}\left(\partial_{I} \widetilde{W}(x)\right)\right|_{x_{3}=x_{4}=0} \\
& \bar{Q}_{2}=\gamma_{a}\left(\partial_{a} \widetilde{W}\right)_{1+\widetilde{\nu}_{a}}=\gamma_{a}\left(\partial_{a i} \widetilde{W}(x)_{-1 / 2}\right) \rho_{i}^{\dagger}=\left.\gamma_{a} \rho_{b}^{\dagger} \partial_{a b} \widetilde{W}(x)\right|_{x_{3}=x_{4}=0}
\end{aligned}
$$

where we have used the moding information, and in the second line also the absence of $\rho_{I}$ excitations at $\bar{q}=-\frac{1}{2}$.

The first of these contributes the following nontrivial $\bar{Q}$ action

$$
\bar{Q}_{1} x_{I} \gamma_{3} \gamma_{4} \bar{\gamma}_{J}|9\rangle=\left.x_{I}\left(\partial_{J} \widetilde{W}(x)\right)\right|_{x_{3}=x_{4}=0} \gamma_{3} \gamma_{4}|9\rangle
$$

so that the cokernel agrees with 103 . When $\widetilde{W}$ is in Fermat form $\bar{Q}_{2}=0$ and there is a six-dimensional space of antichiral singlets at $\bar{q}=\frac{1}{2}$, leaving 19 chiral singlets at $\bar{q}=-\frac{1}{2}$. 


$$
\begin{aligned}
& \bar{q}=-\frac{3}{2} \quad \bar{q}=-\frac{1}{2} \quad \bar{q}=\frac{1}{2} \\
& \gamma_{a} \bar{\gamma}_{b}|1\rangle_{4}
\end{aligned}
$$

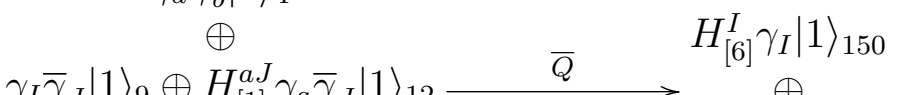

$$
\begin{aligned}
& \gamma_{I} \bar{\gamma}_{J}|1\rangle_{9} \oplus H_{[1]}^{a J} \gamma_{a} \bar{\gamma}_{J}|1\rangle_{12} \longrightarrow \frac{\bar{Q}}{\oplus} \underset{H_{[7]}^{a} \gamma_{a}|1\rangle_{140}}{\oplus} \\
& G_{[2]}^{I} \rho_{I}|1\rangle_{18} \oplus G_{[1]}^{a} \rho_{a}|1\rangle_{4} \\
& K_{[2]}^{I} \bar{\gamma}_{I}|3\rangle_{18} \\
& \oplus \\
& K_{[1]}^{a} \bar{\gamma}_{a}|3\rangle_{4} \\
& \rho_{I}|5\rangle_{3} \\
& \rho_{I} \rho_{J} \rho_{K} \rho_{L} \bar{\gamma}_{3} \bar{\gamma}_{4}|7\rangle_{15} \longrightarrow \bar{Q} \longrightarrow \begin{array}{c}
\rho_{I} \gamma_{J} \bar{\gamma}_{3} \bar{\gamma}_{4}|7\rangle_{9} \oplus x_{a} \rho_{J} \bar{\gamma}_{b}|7\rangle_{12} \\
\rho_{a} \bar{\gamma}_{b}|7\rangle_{4} \oplus \bar{\gamma}_{I}|7\rangle_{3}
\end{array} \\
& x_{I} \gamma_{3} \gamma_{4} \bar{\gamma}_{J}|9\rangle_{9} \oplus x_{I} \rho_{a} \gamma_{b}|9\rangle_{12} \longrightarrow \bar{Q} \longrightarrow F_{[4]} \gamma_{3} \gamma_{4}|9\rangle_{15} \\
& x_{a} \gamma_{b}|9\rangle_{4} \oplus \gamma_{I}|9\rangle_{3} \\
& x_{I}|11\rangle_{3}
\end{aligned}
$$

Table 4: Landau-Ginzburg states for the octic.

When we deform $\widetilde{W}$ away from the Fermat form the cokernel can decrease. For instance, adding the term

$$
\delta \widetilde{W}=\psi x_{0} x_{1} x_{2} x_{3} x_{4}
$$

leaves $\bar{Q}_{1}$ unchanged but adds

$$
\delta \bar{Q}_{2}=\psi\left(\gamma_{3} \rho_{4}^{\dagger}+\gamma_{4} \rho_{3}^{\dagger}\right) x_{0} x_{1} x_{2}
$$

which now acts nontrivially:

$$
\delta \bar{Q}_{2} x_{I} \rho_{a} \gamma_{b}|9\rangle= \pm \psi x_{I} \delta_{a b} x_{0} x_{1} x_{2} \gamma_{3} \gamma_{4}|9\rangle .
$$

The three-dimensional image of this is clearly independent of the image of $\bar{Q}_{1}$ so this deformation removes three additional pairs of states, leaving 16 chiral and 3 antichiral singlets at $k=9$. For generic $\widetilde{W}, \bar{Q}$ has no cokernel and the singlet spectrum is simply 13 chiral states. 
To see that this is identica ${ }^{13}$ to the calculation in the appendix note that in general

$$
\begin{aligned}
\bar{Q}_{2} x_{I} \rho_{a} \gamma_{b}|9\rangle & =\left.\epsilon_{a b} x_{I}\left(\partial_{34} \widetilde{W}\right)\right|_{x_{3}=x_{4}=0} \gamma_{3} \gamma_{4}|9\rangle \\
& =x_{I} \epsilon_{a b}\left(\partial_{5} W\right)_{x_{5}=0} \gamma_{3} \gamma_{4}|9\rangle
\end{aligned}
$$

using the gauge invariance of $W$.

The most obvious physical explanation for this lifting of states is via a simple mass term in the superpotential of the effective theory. The (anti)chiral states in $k=7$ have their chiral(antichiral) conjugates in the $k=9$ sector, allowing for a mass term that depends on the untwisted complex structure moduli.

To summarize, we find $282=2+86+188+6$ singlets for generic $W$, while the Fermat point has an additional $4+12$ massless singlets. This agrees with the Gepner model value of 298 .

\subsubsection{Mirror Symmetry and Kähler Deformations}

The singlet spectrum at the Gepner point in this model includes, in addition to the "cascade states" which as expected are not lifted by Kähler deformations, and the expected four additional singlets lifted by the Higgs mechanism under any deformation, a total of 28 additional chiral singlets in the sectors $k=5,7,9$. The Gepner model here is $A_{3}^{\oplus 3} \oplus A_{7}^{\oplus 2}$. The model enjoys a discrete permutation symmetry $S_{3} \times \mathbb{Z}_{2}$ and the states form representations of this. This symmetry commutes with the quantum symmetry associated to the Gepner orbifold so that states within each twisted sector transform into each other. As always, orbits under the discrete symmetry are lifted together under the Kähler deformations away from the Gepner point, both of which are invariant.

Explicitly, the 28 states comprise eight orbits of the discrete symmetry as listed below. The number in brackets indicates the size of the associated orbit. The 3 chiral states in the $k=5$ sector form the orbit of

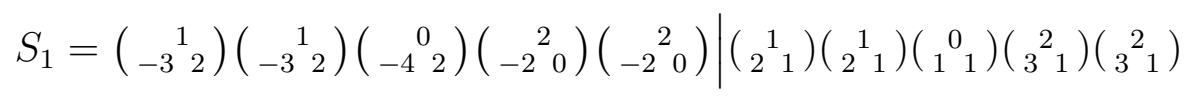

The 6 chiral states in the $k=7$ sector comprise the orbits of

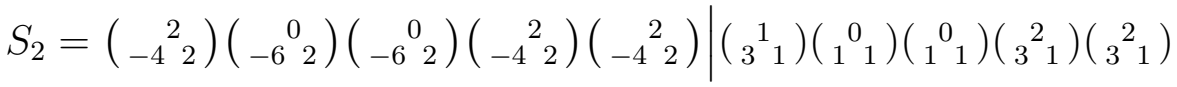

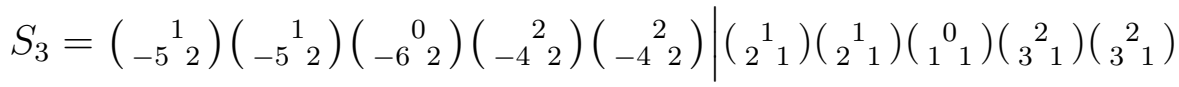

\footnotetext{
${ }^{13}$ Assuming the higher differential $d_{5}$ in the appendix always vanishes.
} 


\begin{tabular}{|c|c|c|}
\hline$\left(k ; t_{1}, t_{2} . t_{3}\right)$ & Orbits & Singlets at Gepner point \\
\hline$(1 ; 0,0,0)$ & $7[2]$ & 7 \\
\hline$(1 ; 1,0,0)$ & 4 & 3 \\
\hline$(1 ; 3,3,2)$ & 1 & 1 \\
\hline$(3 ; 3,3,3)$ & 6 & 2 \\
\hline$(13 ; 3,2,2)$ & 4 & 3 \\
\hline$(15 ; 0,2,2)$ & 2 & 1 \\
\hline$(15 ; 2,1,1)$ & $5 ; 8[2]$ & 3 \\
\hline$(15 ; 3,3,2)$ & 3 & 3 \\
\hline
\end{tabular}

Table 5: Twisted Sectors $(k ; t)$ in which non-cascade singlets arise

The 19 chiral states in the $k=9$ sector comprise the orbits of

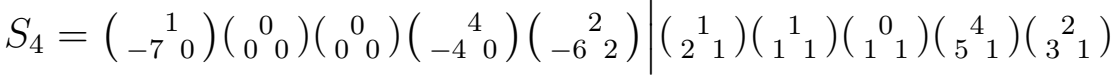

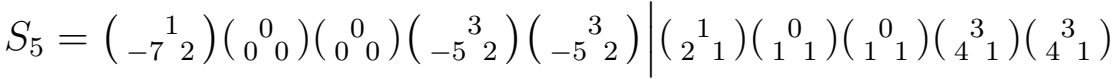

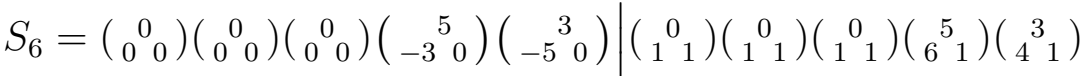

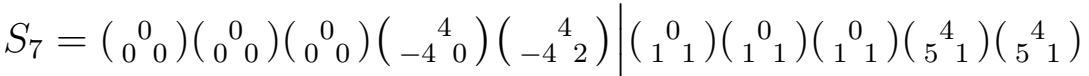

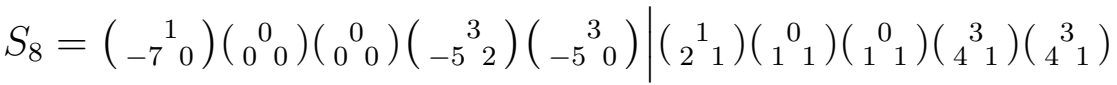

The mirror is given by a $\mathbb{Z}_{4}^{3}$ orbifold, with

$$
m^{(1)}=(1,0,0,0,-2) \quad m^{(2)}=(0,1,0,0,-2) \quad m^{(3)}=(0,0,1,0,-2) .
$$

Table 5.3.4 shows the twisted sectors in which the mirrors of each of these states appear. Note that the $S_{3}$ permutation permutes $t^{(a)}$ while the $\mathbb{Z}_{2}$ acts as $\left(k, t_{a}\right) \mapsto\left(k-4 \sum t_{a}, t_{a}+2 \sum t_{a}\right)$.

The mirror model has two untwisted $(2,2)$ deformations corresponding to the two toric Kähler deformations of the octic. We can write the general superpotential for the mirror model as

$$
\widehat{W}=x_{0}^{4}+x_{1}^{4}+x_{2}^{4}+x_{3}^{8}+x_{4}^{8}-8 \psi x_{0} x_{1} x_{2} x_{3} x_{4}-4 \chi x_{3}^{4} x_{4}^{4} .
$$

In each of the sectors in table 5.3.4 sectors we have computed the $\bar{Q}$ cohomology and the only one in which this changes when we deform $\widehat{W}$ is the sector $(15 ; 2,1,1)$ (and its three permutations). In this sector we have

$$
\nu=\left(\frac{3}{8}, \frac{1}{8}, \frac{1}{8}, \frac{15}{16}, \frac{15}{16}\right) ; \quad \widetilde{\nu}=\left(-\frac{1}{8},-\frac{3}{8},-\frac{3}{8},-\frac{9}{16},-\frac{9}{16}\right)
$$


and a basis for the zero energy states at $q=0$ is

$$
\begin{aligned}
& \bar{q}=-\frac{1}{2} \quad \bar{q}=\frac{1}{2}
\end{aligned}
$$

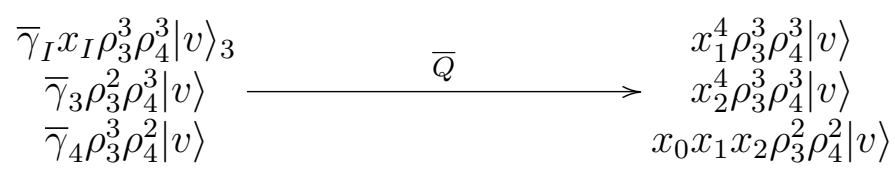

where $|v\rangle$ is the twisted vacuum.

The map $\bar{Q}$ is here given by the terms $\bar{Q}=\bar{\gamma}_{i}^{\dagger}\left(\partial_{i} \widehat{W}\right)_{\widetilde{\nu}_{i}}$ and inserting 129 we compute for our explicit superpotential

$$
\begin{aligned}
\left(\partial_{0} \widehat{W}\right)_{-1 / 8} & =12 x_{0}^{2} \rho_{0}^{\dagger} \\
\left(\partial_{1} \widehat{W}\right)_{-3 / 8} & =4 x_{1}^{3} \\
\left(\partial_{2} \widehat{W}\right)_{-3 / 8} & =4 x_{2}^{3} \\
\left(\partial_{3} \widehat{W}\right)_{-9 / 16} & =8 ! x_{3} \rho_{3}^{\dagger 6}-8 \psi\left(x_{1} x_{2} x_{4} \rho_{0}^{\dagger}+x_{0} x_{2} x_{4} \rho_{1}^{\dagger}+x_{0} x_{1} x_{4} \rho_{2}^{\dagger}+x_{0} x_{1} x_{2} \rho_{4}^{\dagger}\right) \\
& -4(4 !)^{2} \chi \rho_{3}^{\dagger 2} \rho_{4}^{\dagger 3}\left(x_{3} \rho_{4}^{\dagger}+x_{4} \rho_{3}^{\dagger}\right) \\
\left(\partial_{4} \widehat{W}\right)_{-9 / 16} & =8 ! x_{4} \rho_{4}^{\dagger 6}-8 \psi\left(x_{1} x_{2} x_{3} \rho_{0}^{\dagger}+x_{0} x_{2} x_{3} \rho_{1}^{\dagger}+x_{0} x_{1} x_{3} \rho_{2}^{\dagger}+x_{0} x_{1} x_{2} \rho_{3}^{\dagger}\right) \\
& -4(4 !)^{2} \chi \rho_{3}^{\dagger 3} \rho_{4}^{\dagger 2}\left(x_{3} \rho_{4}^{\dagger}+x_{4} \rho_{3}^{\dagger}\right)
\end{aligned}
$$

At the Gepner point $(\psi=\chi=0)$ we have

$$
\bar{Q} \bar{\gamma}_{1} x_{1} \rho_{3}^{3} \rho_{4}^{3}|v\rangle=4 x_{1}^{4} \rho_{3}^{3} \rho_{4}^{3}|v\rangle \quad \bar{Q} \bar{\gamma}_{2} x_{2} \rho_{3}^{3} \rho_{4}^{3}|v\rangle=4 x_{2}^{4} \rho_{3}^{3} \rho_{4}^{3}|v\rangle
$$

as the only nontrivial $\bar{Q}$ action. This leaves 3 chiral singlet states in this sector at the Gepner point, as observed. When we deform, we find that in addition

$$
\bar{Q} \bar{\gamma}_{3} \rho_{3}^{2} \rho_{4}^{3}|v\rangle=\bar{Q} \bar{\gamma}_{4} \rho_{3}^{3} \rho_{4}^{2}|v\rangle=-8 \psi x_{0} x_{1} x_{2} \rho_{3}^{2} \rho_{4}^{2}|v\rangle
$$

Thus, in this sector (and in each of the two related to it by permutations) as well as its conjugate sector (and its permutations) one chiral state is lifted by the $\psi$ deformation. This agrees with the counting above.

Let us conclude the analysis of the octic with a summary of the counting of the singlets. The Gepner model has 298 singlets which may be cataloged as in table 6. In this table we label states "twisted" or "untwisted" according to the orbifold picture of section 5.3.2. 


\begin{tabular}{|c|r|}
\hline Kähler form, untwisted & 1 \\
\hline Kähler form, twisted & 1 \\
\hline Complex Structure, untwisted & 83 \\
\hline Complex Structure, twisted & 3 \\
\hline$H^{1}(\operatorname{End}(T))$ generic untwisted & 182 \\
\hline$H^{1}(\operatorname{End}(T))$ generic twisted & 6 \\
\hline Extra singlets at special complex structure & 12 \\
\hline Extra singlets at special Kähler form & 6 \\
\hline Extra singlets associated to Gepner $\mathrm{U}(1)^{4}$ & 4 \\
\hline \hline Total & 298 \\
\hline
\end{tabular}

Table 6: Classification of the singlets in the Gepner model.

\section{Acknowledgments}

We wish to thank G. Smith and S. Mapes for access to a preview version of [34]. IVM is supported in part by the German-Israeli Project cooperation (DIP H.52) and the GermanIsraeli Fund (GIF). He would also like to thank B. Wurm for enlightening discussions and the CGTP for hospitality while some of this work was being completed. We also thank BIRS and the organizers of the workshop on $(0,2)$ mirror symmetry for providing a great opportunity to begin our elephant exploration. This work was partially supported by NSF grants DMS0606578 and DMS-0905923. Any opinions, findings, and conclusions or recommendations expressed in this material are those of the authors and do not necessarily reflect the views of the National Science Foundation.

\section{A Spectral Sequence Computations}

\section{A.1 Definitions}

Let us first review how to compute the cohomology of a line bundle $\mathscr{O}(\mathbf{q})$ on a toric variety $V$. We need to know this in some detail in order to be able to compute the necessary differentials in the spectral sequence in section 3.2. The method, using local cohomology, is generalized from Grothendieck [45] in [46]. As we describe it, this method is not completely optimized for efficiency, but for our purposes it is not only the actual computation of $H^{k}(V, \mathscr{O}(\mathbf{q}))$ which is important, but also the explicit form of cochains which represents it.

We wish to compute sheaf cohomology in terms of Cech cohomology. The reasoning is exactly analogous to the case of projective space as discussed in [47] chapter III.5. Let the 
irrelevant ideal be $B=\left(m_{1}, m_{2}, \ldots, m_{l}\right)$, where $m_{i}$ are monomials. Consider the open sets

$$
U_{i}=V-Z\left(m_{i}\right) .
$$

These open sets form an open cover of $V$.

The coordinate ring of $U_{i}$ is given by the localization $R_{m_{i}}$ consisting of functions $f / m_{i}^{n}$ for $f \in R$. Similarly $R_{m_{i}, m_{j}}=\left(R_{m_{i}}\right)_{m_{j}}$ is the coordinate ring on $U_{i j}=U_{i} \cap U_{j}$. We then form the local cochain complex from

$$
C_{B}^{i}(R)=\bigoplus_{j_{1}<\ldots<j_{i}} R_{m_{j_{1}}, \ldots, m_{j_{i}}} .
$$

The differential $d: C_{B}^{i}(R) \rightarrow C_{B}^{i+1}(R)$ is given by the obvious inclusion map with some $(-1)^{j}$ factors to ensure $d^{2}=0$. We will elucidate these signs below.

$R$ admits a fine grading valued in $\mathbb{Z}^{n}$, where the grade of each homogeneous coordinate is a basis vector. Let a subscript $p$ denote this fine grading. Then [46]

$$
C_{B}^{*}(R)_{p}=\underset{\left\{J \mid \operatorname{neg}(p) \subset \operatorname{supp}\left(m_{J}\right)\right\}}{\bigoplus} \mathbb{C}
$$

where $J$ is a subset of $\{1, \ldots, l\}, m_{J}$ is the least common multiple of $m_{j}, j \in J$ and $\operatorname{neg}(p)$ is the subset of $\{1, \ldots, l\}$ corresponding to negative grades. The differential $d$ maps the $J$ component of $C_{B}^{*}(R)_{p}$ to the $J^{\prime}$ component as zero unless $J^{\prime}=J \cup j$, in which case it is $(-1)^{e}$ where $j$ is in the eth position of $J^{\prime}$. The local cohomology groups $H_{B}^{i}(R)_{p}$ are defined by the local cochain complex $C_{B}^{*}(R)_{p}$. Note, in particular, that they only depend on $p$ via $\operatorname{neg}(p)$.

The definition of Čech cohomology then gives

$$
\check{C}^{i}\left(\left\{U_{i}\right\}, \mathscr{O}\right)_{p}=C_{B}^{i+1}(R)_{p}, \quad \text { for } i \geq 0 .
$$

We also have $C_{B}^{0}(R)_{p}=R_{p}$. It is a fact that the covering given by $U_{i}$ is affine and thus sufficiently fine to give Cech cohomology. It follows that

$$
H^{i}(V, \mathscr{O})_{p}=H_{B}^{i+1}(R)_{p}, \quad \text { for } i \geq 1,
$$

and that

$$
\begin{aligned}
H^{0}(V, \mathscr{O})_{p} & =R_{p} \\
H_{B}^{0}(R) & =H_{B}^{1}(R)=0 .
\end{aligned}
$$

We now have an explicit method of computing the spectral sequence in theorem 2 . Following the notation of [48, it is based on a double complex $K^{p, q}$, where the vector spaces $K^{p, q}$ have localized Laurent monomials as a basis. The vertical maps in the double complex are the Čech boundaries as explained above, and the horizontal maps come from the complex representing the sheaf in question. 


\section{A.2 The example}

Let $V$ be the resolved weighted projective space $\mathbb{P}_{\{2,2,2,1,1\}}^{4}$. Here $R=\mathbb{C}\left[x_{0}, \ldots, x_{5}\right]$ and $B=\left(x_{0}, x_{1}, x_{2}, x_{5}\right)\left(x_{3}, x_{4}\right)=\left(x_{0} x_{3}, x_{1} x_{3}, x_{2} x_{3}, x_{5} x_{3}, x_{0} x_{4}, x_{1} x_{4}, x_{2} x_{4}, x_{5} x_{4}\right)$. Let $t$ denote the fine grading $(0,0,0,-1,-1,0)$. Then $C_{B}^{*}(R)_{t}$ is given by (starting at position zero)

$$
0 \longrightarrow 0 \longrightarrow \mathbb{C}^{16} \longrightarrow \mathbb{C}^{48} \longrightarrow \mathbb{C}^{68} \longrightarrow \mathbb{C}^{56} \longrightarrow \mathbb{C}^{28} \longrightarrow \mathbb{C}^{8} \longrightarrow \mathbb{C}^{1} .
$$

It is exact in every place except $H_{B}^{2}(R)_{t}=\mathbb{C}$. A generator of $H_{B}^{2}(R)_{t}$ is given by the same Laurent monomial $x_{3}^{-1} x_{4}^{-1}$ in the 16 localizations $R_{m_{i}, m_{j}}$, where $i=0, \ldots 3$ and $j=4 \ldots 7$. One can also show ${ }^{14}$ that $H_{B}^{2}(R)_{p}$ vanishes unless neg $(p)=\operatorname{neg}(t)$.

We now have enough information to compute $H^{1}(V, \mathscr{O}(\mathbf{q})$ for any $\mathbf{q} \in D$. The map from the fine grading to the coarse grading, $D$, is given by the grades $(100)$, which we repeat here for convenience:

$$
\Phi=\left(\begin{array}{cccccc}
0 & 0 & 0 & 1 & 1 & -2 \\
1 & 1 & 1 & 0 & 0 & 1
\end{array}\right) .
$$

The monomial $x_{3}^{-1} x_{4}^{-1}$ has charge $(-2,0)$, and it follows that $H^{1}(V, \mathscr{O}(-2,0))=\mathbb{C}$. Similarly

- $\operatorname{dim} H^{1}(V, \mathscr{O}(-2,1))=3$ with basis $\left\{x_{0} x_{3}^{-1} x_{4}^{-1}, x_{1} x_{3}^{-1} x_{4}^{-1}, x_{2} x_{3}^{-1} x_{4}^{-1}\right\}$ and

- $\operatorname{dim} H^{1}(V, \mathscr{O}(-3,1))=6$ with basis

$$
\left\{x_{0} x_{3}^{-2} x_{4}^{-1}, x_{0} x_{3}^{-1} x_{4}^{-2}, x_{1} x_{3}^{-2} x_{4}^{-1}, x_{1} x_{3}^{-1} x_{4}^{-2}, x_{2} x_{3}^{-2} x_{4}^{-1}, x_{2} x_{3}^{-1} x_{4}^{-2}\right\} .
$$

The first row of the spectral sequence has, for $d_{1}^{0,1}: E^{0,1} \rightarrow E^{1,1}$ :

$$
\begin{aligned}
& H^{1}\left(\mathscr{O}_{X}\right)^{\oplus 4} \\
& \bigoplus_{i, j} H^{1}\left(\mathscr{O}_{X}\left(\mathbf{q}_{i}-\mathbf{q}_{j}\right)\right) \stackrel{d_{1}^{0,1}}{\longrightarrow} \bigoplus_{i} H^{1}\left(\mathscr{O}_{X}\left(\mathbf{q}_{i}\right)\right)^{\oplus 2} \\
& \oplus \quad \bigoplus_{i} H^{1}\left(\mathscr{O}_{X}\left(\mathbf{Q}-\mathbf{q}_{i}\right)\right) \\
& H^{1}\left(\mathscr{O}_{X}\right)
\end{aligned}
$$

In our case this becomes

$$
\begin{aligned}
& H^{1}(\mathscr{O}(-2,0))^{\oplus 3} \stackrel{\oplus}{d_{1}^{0,1}} \longrightarrow H^{1}(\mathscr{O}(-2,1))^{\oplus 2} . \\
& H^{1}(\mathscr{O}(-3,1))^{\oplus 2}
\end{aligned}
$$

Note that in each case here the restriction of $H^{1}\left(\mathscr{O}_{V}(\mathbf{q})\right)$ to $H^{1}\left(\mathscr{O}_{X}(\mathbf{q})\right)$ is the identity, and so we have dropped the subscript from $\mathscr{O}$.

\footnotetext{
${ }^{14}$ For a quick method see, for example, section 3 of 49 .
} 
The map $d_{1}^{0,1}$ is induced from the map $E$ in 45 , which is given by

$$
\begin{aligned}
& \mathscr{O}^{\oplus 2} \stackrel{\left(\begin{array}{cc}
0 & x_{0} \\
0 & x_{1} \\
0 & x_{2} \\
x_{3} & 0 \\
x_{4} & 0 \\
-2 x_{5} & x_{5}
\end{array}\right)}{\longrightarrow} \mathscr{O}(1,0)^{\oplus 2} . \\
& \oplus \\
& \mathscr{O}(-2,1)
\end{aligned}
$$

Using the Laurent monomial representatives of these cocycles we easily obtain the map $d_{1}^{0,1}$ :

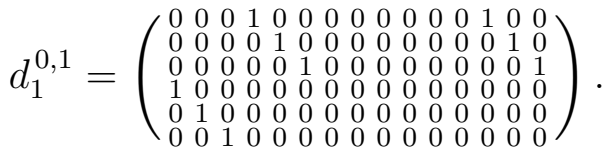

The spectral sequence at the $E_{1}$ stage is given by 102 . Now we have computed all the $d_{1}$ maps, and we obtain

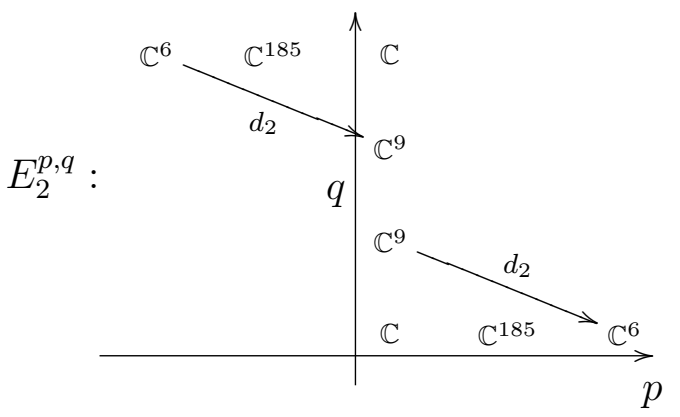

So now we need to compute the $d_{2}$ maps. Fortunately they are related by Serre duality. Let us focus on $d_{2}^{0,1}: \mathbb{C}^{9} \rightarrow \mathbb{C}^{6}$.

The map $d_{2}$ will depend upon the defining equation $W$. Only certain monomials terms will contribute to $d_{2}$. For purposes of argument we concentrate first on a single monomial $x_{0} x_{2}^{2} x_{3}^{2} x_{5}$.

The process of computing $d_{2}$ can be quite formidable in a general spectral sequence, but the explicit representatives of elements of the double complex $K^{p, q}$ as Laurent monomials makes the procedure straight-forward. Consider, as an example, the monomial $x_{0} x_{3}^{-2} x_{4}^{-1}$ representing an element of $H^{1}(\mathscr{O}(-3,1))$ in $K^{0,1}$.

The map to $K^{1,1}$ has two contributions. First we map $\check{C}^{1}(\mathscr{O}(-3,1))$ to $\check{C}^{1}(\mathscr{O}(-2,1)$ by multiplying by $x_{i} \mathbf{q}_{i}$, which in the case of interest amounts to multiplying by $x_{4}$. We also map $\check{C}^{1}(\mathscr{O}(-3,1))$ to $\check{C}^{1}(\mathscr{O}(-1,4))$ by multiplying by $\partial_{5} W$. Thus we map $x_{0} x_{3}^{-2} x_{4}^{-1}$ to

$$
\left(\frac{x_{0}}{x_{3}^{2}}, \frac{x_{0}^{2} x_{2}^{2}}{x_{4}}\right) \in \check{C}^{1}(\mathscr{O}(-2,1)) \oplus \check{C}^{1}(\mathscr{O}(-1,4)) .
$$

This is a Čech coboundary, and we can chase it downwards as follows. Recall that these monomial representatives of $\check{C}^{1}(\mathscr{O}(\mathbf{q}))$ are really 16 copies of the same monomials under the 
localization $R_{m_{i}, m_{j}}$. Computing the chain complex $C_{B}^{*}(R)_{(0,0,0,-1,0,0)}$, we see that $x_{0} x_{3}^{-2}$ lies in the coboundary of the 4 copies of the monomial localized to $R_{m_{i}}$, where $i=0, \ldots, 3$. Similarly $x_{0}^{2} x_{2}^{2} x_{4}^{-1}$ lies in the coboundary of the 4 copies of the negated monomial localized to $R_{m_{j}}$, where $i=4, \ldots, 7$.

Finally we apply $d_{1}$ to push our element to $K^{0,2} \cdot x_{0} x_{3}^{-2}$ is multiplied by $\partial_{5} W$, while $x_{0}^{2} x_{2}^{2} x_{4}^{-1}$ is multiplied by $-x_{4}$. Paying attention to signs, the result is $x_{0}^{2} x_{2}^{2}$ in both cases. That is, we have a Čech cochain which takes the value $x_{0}^{2} x_{2}^{2}$ in all eight patches $R_{m_{k}}$, $k=1, \ldots, 8$.

The fact this has the same value in all 8 patches means that this is the localization of a monomial in $R$ itself. This had to be, since $H_{B}^{1}(R)=0$, and allows us to interpret $x_{0}^{2} x_{2}^{2}$ as an element of $H^{0}(\mathscr{O}(\mathbf{Q}))$. The computation of $d_{2}$ can be summarized in the following diagram:

$$
\begin{aligned}
\left(\frac{x_{0}}{x_{3}^{2} x_{4}}\right)_{i j} \stackrel{\left(\begin{array}{c}
x_{4} \\
x_{0} x_{2}^{2} x_{3}^{2}
\end{array}\right)}{\longrightarrow}\left(\frac{x_{0}}{x_{3}^{2}}\right)_{i j},\left(\frac{x_{0}^{2} x_{2}^{2}}{x_{4}}\right)_{i j} & \\
& \left(\frac{x_{0}}{x_{3}^{2}}\right)_{i},-\left(\frac{x_{0}^{2} x_{2}^{2}}{x_{4}}\right)_{j} \stackrel{\left(x_{0} x_{2}^{2} x_{3}^{2},-x_{4}\right)}{\longrightarrow}\left(\begin{array}{c}
\left.x_{0}^{2} x_{2}^{2}\right)_{k} \\
\left.d\right|_{k} \\
\end{array}\right.
\end{aligned}
$$

Thus, $d_{2}$ is nonzero.

Given a generic defining equation $W$ with all possible monomials, this $d_{2}$ map is surjective and the sequence degenerates at the $E_{3}$ term:

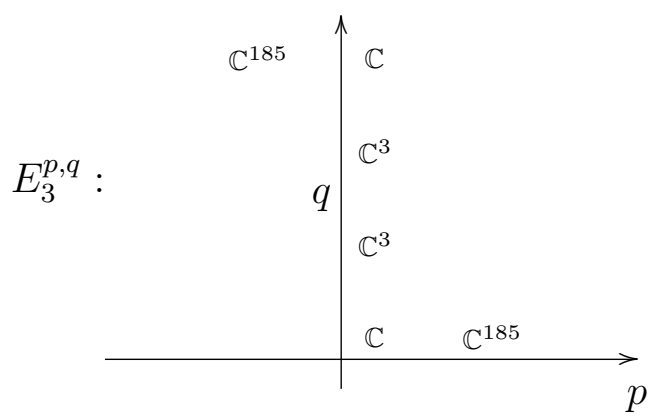

So $\operatorname{dim} H^{1}(X, \operatorname{End}(T))=188$.

However, suppose we pick the Fermat form of the defining equation $W$. Studying the above computation of $d_{2}$, it is clear that we can never hit monomials in $K^{0,2}$ of the form 
$x_{0}^{2} x_{2}^{2}$, etc. Thus, the $d_{2}$ maps are actually zero. Now we go to the $E_{4}$ stage:

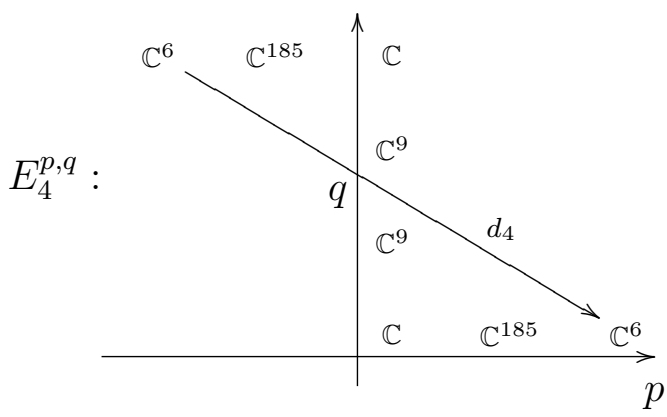

The source of the $d_{4}$ map is a $\mathbb{C}^{6}$ subspace of $H^{3}\left(\mathscr{O}_{X}(-\mathbf{Q})\right)^{\oplus 2}$. This third cohomology group is not the isomorphic image of $H^{3}\left(\mathscr{O}_{V}(-\mathbf{Q})\right)$ under restriction, and so we need to work a little harder to describe everything in terms of cohomology of the toric variety and thus local cohomology. To this end we may use the short exact sequence

$$
0 \longrightarrow \mathscr{O}_{V}(-\mathbf{Q}) \longrightarrow \mathscr{O}_{V} \longrightarrow \mathscr{O}_{X} \longrightarrow 0,
$$

to write $\mathscr{O}_{X}$ in terms of $\mathscr{O}_{V}$. By using mapping cones, we may write the complex (45) representing the tangent sheaf as

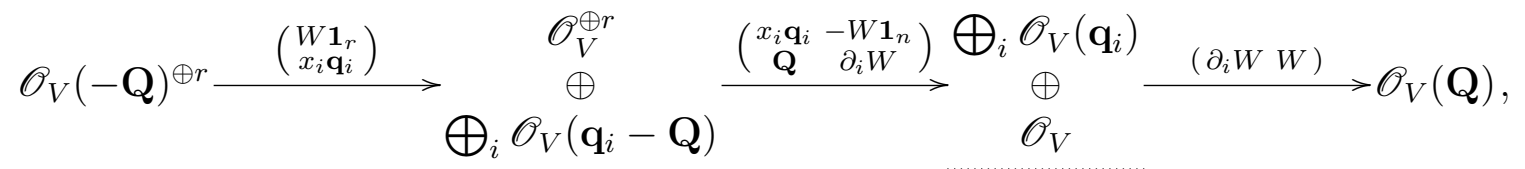

and we can take the $\mathscr{H}$ om of this complex into itself to form a complex for $\operatorname{End}(T)$. The $H^{3}\left(\mathscr{O}_{X}(-\mathbf{Q})\right)^{\oplus 2}$ from above now manifests itself as $H^{4}\left(\mathscr{O}_{V}(-2 \mathbf{Q})\right)^{\oplus 2}$, and the interesting map appears in $E_{5}$ as ${ }^{15}$

$$
d_{5}: H^{4}\left(\mathscr{O}_{V}(-2 \mathbf{Q})\right)^{\oplus 2} \rightarrow H^{0}\left(\mathscr{O}_{V}(\mathbf{Q})\right)^{\oplus 2} .
$$

With a little organization one can show, for example, that the Fermat form of $W$ cannot give rise to a nonzero map ${ }^{16}$ Thus, in this case, the spectral sequence degenerates at the $E_{2}$ stage again, and now $\operatorname{dim} H^{1}(X, \operatorname{End}(T))=185+9+6=200$. That is we have 12 extra states compared to the generic hypersurface.

\section{References}

[1] P. Candelas, G. Horowitz, A. Strominger, and E. Witten, Vacuum Configuration for Superstrings, Nucl. Phys. B258 (1985) 46-74.

\footnotetext{
${ }^{15}$ This map is really only defined on a subspace of the term on the left and a quotient of the space on the right, since we are taking cohomology at the earlier stages of the spectral sequence.

${ }^{16}$ This $d_{5}$ map is a quite tedious to calculate but appears to be zero for any $W$.
} 
[2] T. Banks and L. J. Dixon, Constraints on String Vacua with Space-Time Supersymmetry, Nucl. Phys. B307 (1988) 93-108.

[3] L. J. Dixon, Some World-Sheet Properties of Superstring Compactifications,on Orbifolds and Otherwise, in G. Furlan et al., editors, "Superstrings, Unified Theories, and Cosmology 1987", pages 67-126, World Scientific, 1988.

[4] G. Tian, Smoothness of the Universal Deformation Space of Compact Calabi-Yau Manifolds and its Petersson-Weil Metric, in S.-T. Yau, editor, "Mathematical Aspects of String Theory", pages 629-646, World Scientific, Singapore, 1987.

[5] P. Berglund, T. Hübsch, and L. Parkes, Gauge Neutral Matter in Three Generation Superstring Compactifications, Mod. Phys. Lett. A5 (1990) 1485-1492.

[6] M. Dine, N. Seiberg, X. G. Wen, and E. Witten, Nonperturbative Effects on the String World-Sheet, Nucl. Phys. B278 (1986) 769-789, and Nucl. Phys. B289 (1987) 319-363.

[7] C. A. Lütken and G. G. Ross, Taxonomy of Heterotic Superconformal Field Theories, Phys. Lett. B213 (1988) 152.

[8] E. Witten, Phases of $N=2$ Theories in Two Dimensions, Nucl. Phys. B403 (1993) 159-222, hep-th/9301042.

[9] M. Kreuzer, J. McOrist, I. V. Melnikov, and M. R. Plesser, (0,2) Deformations of Linear Sigma Models, arXiv:1001.2104.

[10] E. Silverstein and E. Witten, Criteria for conformal invariance of (0,2) models, Nucl. Phys. B444 (1995) 161-190, hep-th/9503212.

[11] A. Basu and S. Sethi, World-sheet stability of (0,2) linear sigma models, Phys. Rev. D68 (2003) 025003, hep-th/0303066.

[12] C. Beasley and E. Witten, Residues and world-sheet instantons, JHEP 10 (2003) 065, hep-th/0304115.

[13] L. Dixon, J. A. Harvey, C. Vafa, and E. Witten, Strings on Orbifolds, Nucl. Phys. B261 (1985) 678-686, and B274 (1986) 285-314.

[14] E. Martinec, Algebraic Geometry and Effective Lagrangians, Phys. Lett. 217B (1989) $431-437$.

[15] C. Vafa and N. Warner, Catastrophes and the Classification of Conformal Theories, Phys. Lett 218B (1989) 51-58.

[16] S. Kachru and E. Witten, Computing the complete massless spectrum of a LandauGinzburg orbifold, Nucl. Phys. B407 (1993) 637-666, hep-th/9307038. 
[17] E. Witten, On the Landau-Ginzburg Description of $N=2$ Minimal Models, Int. J. Mod. Phys. A9 (1994) 4783-4800, hep-th/9304026.

[18] J. Distler and S. Kachru, (0,2) Landau-Ginzburg theory, Nucl. Phys. B413 (1994) 213-243, hep-th/9309110.

[19] T. Kawai and K. Mohri, Geometry of (0,2) Landau-Ginzburg orbifolds, Nucl. Phys. B425 (1994) 191-216, hep-th/9402148.

[20] J. Distler, Notes on (0,2) Superconformal Field Theories, hep-th/9502012.

[21] D. Gepner, Space-Time Supersymmetry in Compactified String Theory and Superconformal Models, Nucl. Phys. B296 (1988) 757.

[22] C. Vafa, String Vacua and Orbifoldized L-G Models, Mod. Phys. Lett. A4 (1989) 1169.

[23] K. A. Intriligator and C. Vafa, Landau-Ginzburg Orbifolds, Nucl. Phys. B339 (1990) 95-120.

[24] D. Gepner, Exactly Solvable String Compactifications on Manifolds of SU(N) Holonomy, Phys. Lett. 199B (1987) 380-388.

[25] B. R. Greene and M. R. Plesser, Duality in Calabi-Yau Moduli Space, Nucl. Phys. B338 (1990) 15-37.

[26] M. Green, J. Schwarz, and E. Witten, Superstring Theory, Cambridge University Press, 1987, 2 volumes.

[27] M. G. Eastwood and T. Hübsch, Endomorphism Valued Cohomology and Gauge Neutral Matter, Commun. Math. Phys. 132 (1990) 383-414.

[28] T. Hübsch, Calabi-Yau Manifolds: A Bestiary for Physicists, World Scientific, Singapore, 1992.

[29] V. V. Batyrev, Dual Polyhedra and Mirror Symmetry for Calabi-Yau Hypersurfaces in Toric Varieties, J. Alg. Geom. 3 (1994) 493-535.

[30] V. V. Batyrev and L. A. Borisov, Dual Cones and Mirror Symmetry for Generalized Calabi-Yau Manifolds, in B. Greene and S.-T. Yau, editors, "Mirror Symmetry II", pages 71-86, International Press, 1994, alg-geom/9402002.

[31] D. A. Cox, The Homogeneous Coordinate Ring of a Toric Variety, J. Algebraic Geom. 4 (1995) 17-50, alg-geom/9210008.

[32] V. V. Batyrev and D. A. Cox, On the Hodge Structure of Projective Hypersurfaces in Toric Varieties, Duke Math. J 75 (1994) 293-338, arXiv:alg-geom/9306011.

[33] H. Cartan and S. Eilenberg, Homological algebra, Princeton, 1956. 
[34] G. G. Smith, NormalToric Varieties - A Package for Macaulay 2, to appear in the Macaulay 2 distribution.

[35] N. O. Ilten, Deformations of Smooth Toric Surfaces, arXiv:0902.0529.

[36] S. I. Gelfand and Y. I. Manin, Homological Algebra, Encyclopædia of Mathematical Sciences 38, Springer, 1994.

[37] C. A. Weibel, An Introduction to Homological Algebra, Cambridge Stud. in Adv. Math. 38, Cambridge, 1994.

[38] P. Seidel and R. P. Thomas, Braid Groups Actions on Derived Categories of Coherent Sheaves, Duke Math. J. 108 (2001) 37-108, math.AG/0001043.

[39] E. Witten, New Issues in Manifolds of SU(3) Holonomy, Nucl. Phys. B268 (1986) $79-112$.

[40] P. S. Aspinwall, B. R. Greene, and D. R. Morrison, The Monomial-Divisor Mirror Map, Internat. Math. Res. Notices 1993 319-338, alg-geom/9309007.

[41] P. Candelas, X. de la Ossa, and S. H. Katz, Mirror Symmetry for Calabi-Yau Hypersurfaces in Weighted $\mathbb{P}_{4}$ and Extensions of Landau-Ginzburg Theory, Nucl. Phys. B450 (1995) 267-292, hep-th/9412117.

[42] A. C. Avram, P. Candelas, D. Jančic, and M. Mandelberg, On the Connectedness of Moduli Spaces of Calabi-Yau Manifolds, Nucl. Phys. B465 (1996) 458-472, hepth/9511230.

[43] P. M. H. Wilson, The Kähler Cone on Calabi-Yau Threefolds, Invent. Math. 107 (1992) $561-583$.

[44] P. Candelas et al., Mirror Symmetry for Two Parameter Models - I, Nucl. Phys. B416 (1994) 481-562, hep-th/9308083.

[45] A. Grothendieck, Local Cohomology, Lecture Notes in Math. 41, Springer, 1967.

[46] D. Eisenbud, M. Mustaţă, and M. Stillman, Cohomology on Toric Varieties and Local Cohomology with Monomial Supports, J. Symbolic Comput. 29 (2000) 583-600, arXiv:math/0001159.

[47] R. Hartshorne, Algebraic Geometry, Graduate Texts in Mathematics 52, SpringerVerlag, 1977.

[48] R. Bott and L. W. Tu, Differential Forms in Algebraic Topology, Springer-Verlag, New York, 1982.

[49] M. Mustaţă, Local Cohomology at Monomial Ideals, J. Symbolic Comput. 29 (2000) 709-720, arXiv:math/0001153. 